\title{
The Solar Neighborhood. XLII. Parallax Results from the CTIOPI 0.9 m Program-Identifying New Nearby Subdwarfs Using Tangential Velocities and Locations on the H-R Diagram
}

\author{
Wei-Chun Jao (饒惟君) $)^{1,6}$ (1) Todd J. Henry ${ }^{2,6}$, Jennifer G. Winters ${ }^{3,6}$, John P. Subasavage ${ }^{4,6}$ (D) Adric R. Riedel ${ }^{5,6}$ (D), \\ Michele L. Silverstein ${ }^{1,6}\left(\mathbb{D}\right.$, and Philip A. Ianna ${ }^{2,6}$ \\ ${ }^{1}$ Department of Physics and Astronomy, Georgia State University, Atlanta, GA 30302, USA; jao@astro.gsu.edu, silverstein@astro.gsu.edu \\ ${ }^{2}$ RECONS Institute, Chambersburg, PA 17201, USA; toddhenry28@gmail.com, philianna3@gmail.com \\ ${ }^{3}$ Harvard-Smithsonian Center for Astrophysics, Cambridge, MA 02138, USA; jennifer.winters@cfa.harvard.edu \\ ${ }^{4}$ United States Naval Observatory, Flagstaff Station, Flagstaff, AZ 86601, USA; jsubasavage@nofs.navy.mil \\ ${ }^{5}$ Astronomy Department, California Institute of Technology, Pasadena, CA 91125, USA; arr@ astro.caltech.edu \\ Received 2017 August 9; revised 2017 September 6; accepted 2017 September 6; published 2017 October 20
}

\begin{abstract}
Parallaxes, proper motions, and optical photometry are presented for 51 systems consisting of 37 cool subdwarf and 14 additional high proper motion systems. Thirty-seven systems have parallaxes reported for the first time, 15 of which have proper motions of at least $1^{\prime \prime} \mathrm{yr}^{-1}$. The sample includes 22 newly identified cool subdwarfs within $100 \mathrm{pc}$, of which three are within $25 \mathrm{pc}$, and an additional five subdwarfs from 100 to $160 \mathrm{pc}$. Two systems-LSR 1610-0040 AB and LHS $440 \mathrm{AB}$-are close binaries exhibiting clear astrometric perturbations that will ultimately provide important masses for cool subdwarfs. We use the accurate parallaxes and proper motions provided here, combined with additional data from our program and others, to determine that effectively all nearby stars with tangential velocities greater than $200 \mathrm{~km} \mathrm{~s}^{-1}$ are subdwarfs. We compare a sample of 167 confirmed cool subdwarfs to nearby main sequence dwarfs and Pleiades members on an observational Hertzsprung-Russell diagram using $M_{V}$ versus $\left(V-K_{s}\right)$ to map trends of age and metallicity. We find that subdwarfs are clearly separated for spectral types K5-M5, indicating that the low metallicities of subdwarfs set them apart in the H-R diagram for $\left(V-K_{s}\right)=3-6$. We then apply the tangential velocity cutoff and the subdwarf region of the H-R diagram to stars with parallaxes from Gaia Data Release 1 and the MEarth Project to identify a total of 29 new nearby subdwarf candidates that fall clearly below the main sequence.
\end{abstract}

Key words: astrometry - solar neighborhood - stars: distances - stars: low-mass - subdwarfs

Supporting material: machine-readable tables

\section{Introduction}

Cool subdwarfs of spectral types G, K, and M are Galactic relics with relatively low metallicities compared to their dwarf counterparts (Chamberlain \& Aller 1951; Mould 1976). Unlike the abundant metal-rich dwarfs in the solar neighborhood, there are currently only three confirmed subdwarf systems within $10 \mathrm{pc}$ : $\mu$ Cas AB, CF UMa, and Kapteyn's Star (Monteiro et al. 2006), making them a minority in our solar neighborhood. Because of their scarcity and intrinsic faintness, fewer key stellar parameters, such as radius and mass, have been measured for subdwarfs compared to the dwarfs. For example, Jao et al. (2016) showed that there are only five nearby confirmed subdwarf binaries with measured dynamical masses, compared to at least five times that for M dwarf dynamical masses alone (Henry et al. 1999; Benedict et al. 2016). Direct measurements of stellar radii are almost entirely for main sequence dwarfs (Ségransan et al. 2003; Berger et al. 2006; López-Morales 2007; Torres et al. 2010; Boyajian et al. 2012). The $\mu$ Cas $A$ is the only subdwarf ${ }^{7}$ with

\footnotetext{
${ }^{6}$ Visiting Astronomer, Cerro Tololo Inter-American Observatory. CTIO is operated by AURA, Inc. under contract to the National Science Foundation.

7 A compendium of eclipsing binaries by López-Morales (2007) and Torres et al. (2010) shows a few stars with $[\mathrm{Fe} / \mathrm{H}] \leqslant-0.5$, but almost all of them are early type subdwarfs. Of particular note, GJ 630.1 AB (CM Dra AB) is an eclipsing binary with $[\mathrm{Fe} / \mathrm{H}]=-0.67$ (López-Morales 2007), but Hawley et al. (1996) assigned it spectroscopically as a M4.5 V, i.e., a main sequence star. Furthermore, a wide third component is a white dwarf with an estimated age of $3 \mathrm{Gyr}$ (Bergeron et al. 1997), so it is not old enough to be a subdwarf. This shows inconsistent results between metallicity, spectral classification, and ages. Hence, we do not consider GJ 630.1 AB to be a subdwarf system.
}

interferometric measurement of its radius (Boyajian et al. 2008), but it is a G-type subdwarf. Thus, to understand the nature of the metal-poor stars that formed early in the history of the Galaxy, it is important to identify more nearby subdwarfs so that the most basic stellar parameters of masses and radii can be determined.

In order to reveal nearby subdwarfs, the Cerro Tololo Interamerican Observatory Parallax Investigation (CTIOPI) carried out by RECONS (REsearch Consortium On Nearby Stars) ${ }^{8}$ has targeted subdwarf candidates with high proper motions extracted from various catalogs and surveys (Giclas et al. 1971; Giclas 1979; Luyten 1979; Pokorny et al. 2003; Hambly et al. 2004; Scholz et al. 2004a; Deacon et al. 2005; Lépine \& Shara 2005; Gizis et al. 2011). Here, we present the first parallaxes for 37 stellar systems selected from these surveys and revised parallaxes for 14 additional systems. As in previous papers in The Solar Neighborhood series, the overlap in the samples of fast-moving and low-metallicity stars makes it natural to combine the two types of objects in this paper with the primary goal of unveiling more nearby missing subdwarfs.

\section{Observations and Data Reduction}

We used the CTIO/SMARTS $0.9 \mathrm{~m}$ to measure parallaxes and optical photometry in the VRI filters. The telescope has a $2048 \times 2046$ Tektronix CCD camera with 0"' 401 pixel $^{-1}$ plate scale (Jao et al. 2005). For both astrometric and photometric observations, we used the central quarter of the chip, yielding a

http://www.recons.org 
6'.8 square field of view. We used the Johnson $V$ and KronCousins $R I$ filters for parallax measurements to maximize the number of suitable reference stars in the field; because of their relative faintness, 31 of the 51 systems were observed in the $I$ band. For the 51 systems discussed here, astrometric series spanned 2-15 years with a median of 5 years. We also obtained VRI photometry of the targets and parallax reference stars through the same filters. The photometry was used to characterize the stars, remove differential color refraction offsets in the astrometry, and correct the relative parallaxes to absolute parallaxes via photometric distance estimates of the reference stars.

Bias and dome flat frames were taken nightly for basic image reductions and calibrations. Details of our observing methodology and data reduction, including astrometric, photometric, and spectroscopic techniques, have been discussed in previous parallax papers of The Solar Neighborhood series; in particular, see (Jao et al. 2005) for astrometry protocols and (Winters et al. 2015) for photometry methods.

\section{Results}

\subsection{Astrometry Results}

The astrometry results are presented in Table 1, where we provide details about the astrometric observations. The first column gives the target identifiers, followed by coordinates (column 2), filters used (3), number of seasons observed (4), number of frames used in reductions (5), time coverage (6), the total time spans (7), the number of reference stars (8), relative parallaxes (9), parallax corrections (10), absolute parallaxes (11), proper motions (12), position angles of the proper motions (13), and the derived tangential velocities (14). An exclamation point in the Note column indicates that additional details about that system are provided in Section 4.

High proper motion stars fall into two astrophysically interesting categories - nearby stars and those with intrinsically high space velocities, typically subdwarfs. Among the 37 systems for which we provide the first parallaxes here, 15 are moving faster than $1^{\prime \prime} \mathrm{yr}^{-1}$, including seven subdwarfs, seven main sequence red dwarfs, and a brown dwarf 2MA $1506+1321$.

Parallax errors are less than 2 mas for all but five systems. Among the 37 systems with parallaxes reported for the first time here, nine are within 25 parsecs (pc) and nine more are between 25 and $60 \mathrm{pc}$. The latter horizon is being used to build a volume-complete sample of the nearest cool subdwarfs, and includes nine new subdwarfs first identified to be within $60 \mathrm{pc}$ here-LEHPM 1-4592, LHS 1257, LHS 1490, LHS 2096, LHS 2099, LHS 2140, LHS 2904, LSR 0609+2319, and SSS 1358-3938. The remaining 19 systems are between 60 and 160 pc. Overall, the RECONS astrometry program on both the $0.9 \mathrm{~m}$ and $1.5 \mathrm{~m}$ at CTIO have added 26 new cool subdwarfs within 60 pc (Costa et al. 2005; Jao et al. 2005, 2011) since 1999, including this work. This constitutes a significant increase of $25 \%$ to the previously known sample (van Altena et al. 1995; Perryman et al. 1997; Burgasser et al. 2008; Schilbach et al. 2009; Smart et al. 2010). ${ }^{9}$

\footnotetext{
9 A comprehensive discussion of the entire $60 \mathrm{pc}$ cool subdwarf sample is planned for a future paper in this series.
}

\subsection{Photometry Results}

Results of our VRI photometry, as well as the near-IR photometry from the Two Micron All Sky Survey (Skrutskie et al. 2006), are presented in Table 2. The first two columns provide identifiers, followed by the VRI magnitudes (columns 3,4 , and 5), the number of $V R I$ observations (6), the filter in which parallax frames were taken (7), the variability in that filter (8), the $J H K_{s}$ photometry $(9,10$, and 11), the spectral type (12), and the spectral type reference (13).

Stars were observed in VRI filters, spanning magnitude ranges of $V=11.49-20.25, R=10.49-19.30$, and $I=9.31-$ 18.34. All stars were observed in all three filters except 2MA1506+1321, which is too faint in $V$ to be observed effectively at the $0.9 \mathrm{~m}$ telescope, but for which $R$ and $I$ magnitudes are provided. All stars except SIP 1540-2613 were observed 2-4 times. As described in detail in Winters et al. (2011), the mean standard deviations of our multi-epoch photometry are typically $\sim 0.03 \mathrm{mag}$ in $V$ and $\sim 0.02 \mathrm{mag}$ in $R$ and $I$ bands. This is true regardless of magnitude, as fainter stars are simply observed with longer integrations to increase signal-to-noise.

The combination of our astrometry and photometry results allows us to place the sample of stars on the observational $\mathrm{H}-\mathrm{R}$ diagram shown in Figure 1, which uses $M_{V}$ and $\left(V-K_{S}\right)$. Stars within $25 \mathrm{pc}$ are represented with gray points, overlaid with the sample stars in black. Several noteworthy stars discussed in Section 4 are circled and labeled in red.

\subsection{Variability Results}

The long-term data series of images taken for astrometry of the observed stars permits an evaluation of their photometric variability in the filter used for the observations. Listed in column 8 of Table 2 are the variability results for each target.

Jao et al. (2011) first reported the long-term variability of our parallax stars with coverage from 2-10 years and found that the 22 cool subdwarfs investigated at the time were substantially less photometrically variable than the 108 main sequence red dwarf examined. Hosey et al. (2015) then expanded the variability study to $264 \mathrm{M}$ dwarfs and found that only $8 \%$ of $\mathrm{M}$ dwarfs are photometrically variable by at least $20 \mathrm{mmag}$. Details of the data-reduction processes used to determine variability can be found in those two papers. The median variability of the 42 subdwarfs in this work is only $9 \mathrm{mmag}$, with only one subdwarf, LHS2852 (24 mmag), having a variability greater than $20 \mathrm{mmag}$. Hence, we reconfirm the conclusion we made in Jao et al. (2011) that subdwarfs are, in general, photometrically quiet. We note, however, that because of the faintness of the targets in this sample, most (26 of 42) of the subdwarfs were observed in the $I$ band for parallax observations, so the variability for those objects is likely to be lower than stars observed in the $V$ band, which includes potentially variable $\mathrm{H} \alpha$ emission.

\subsection{Spectroscopy of Cool Subdwarfs}

Spectral types from the literature, including many of our own results, are given in columns 12 and 13 of Table 2. Although we do not present any new spectra in this paper, for stars with no spectra available, we can make informed estimates of luminosity classes based on the astrometry and photometry data presented here, as discussed in Sections 3.1 and 3.2. We assign a luminosity class of "VI" to 14 stars we now identify to be 
Table 1

Astrometry Results

\begin{tabular}{|c|c|c|c|c|c|c|c|c|c|c|c|c|c|c|c|}
\hline Name & $\begin{array}{l}\text { R.A. } \\
\text { (J20 }\end{array}$ & 00.0) & Filt & Nsea & Nfrm & Coverage & Years & Nref & $\begin{array}{c}\pi(\mathrm{rel}) \\
(\mathrm{mas}) \\
(9)\end{array}$ & $\begin{array}{c}\pi \text { (corr) } \\
(\text { mas }) \\
(10)\end{array}$ & $\begin{array}{c}\pi(\mathrm{abs}) \\
(\mathrm{mas}) \\
(11)\end{array}$ & $\begin{array}{c}\mu \\
\left(\operatorname{mas~yr}^{-1}\right) \\
(12)\end{array}$ & $\begin{array}{l}\text { P.A. } \\
\text { (deg) } \\
(13)\end{array}$ & $\begin{array}{c}V_{\tan } \\
\left(\mathrm{km} \mathrm{s}^{-1}\right) \\
(14)\end{array}$ & Note \\
\hline \multicolumn{16}{|c|}{ First Trigonometric Parallaxes } \\
\hline LHS 1048 & 001533.51 & -351147.6 & $I$ & $8 \mathrm{~s}$ & 69 & 2005.71-2012.89 & 7.18 & 8 & $24.47 \pm 1.06$ & $1.64 \pm 0.16$ & $26.11 \pm 1.07$ & $949.4 \pm 0.4$ & $100.1 \pm 0.04$ & 172.4 & \\
\hline LHS 127 & 005543.89 & -211307.1 & $I$ & $8 \mathrm{~s}$ & 61 & 2003.94-2012.94 & 8.99 & 7 & $14.98 \pm 1.10$ & $0.52 \pm 0.03$ & $15.50 \pm 1.10$ & $1227.7 \pm 0.4$ & $99.2 \pm 0.03$ & 375.6 & \\
\hline LEHPM $1-1628$ & 013105.40 & -502510.0 & $I$ & $7 \mathrm{~s}$ & 50 & 2005.72-2012.94 & 7.22 & 9 & $10.05 \pm 1.83$ & $0.50 \pm 0.06$ & $10.55 \pm 1.83$ & $1083.0 \pm 0.6$ & $142.1 \pm 0.06$ & 486.5 & \\
\hline LHS 1257 & 013130.82 & +100129.7 & I & $7 \mathrm{~s}$ & 48 & $2005.80-2012.81$ & 7.01 & 7 & $20.00 \pm 2.25$ & $0.96 \pm 0.13$ & $20.96 \pm 2.25$ & $929.6 \pm 0.7$ & $158.1 \pm 0.08$ & 210.2 & \\
\hline LHS 150 & 020723.26 & -663411.6 & $V$ & $9 \mathrm{~s}$ & 69 & 2003.95-2012.70 & 8.75 & 8 & $84.91 \pm 1.83$ & $1.24 \pm 0.12$ & $86.15 \pm 1.83$ & $1774.2 \pm 0.7$ & $78.2 \pm 0.04$ & 97.6 & \\
\hline LHS 1490 & 030206.36 & -395051.9 & $I$ & $8 \mathrm{~s}$ & 87 & 2007.55-2015.96 & 8.41 & 9 & $70.46 \pm 1.62$ & $0.31 \pm 0.02$ & $70.77 \pm 1.62$ & $850.6 \pm 0.8$ & $220.6 \pm 0.11$ & 57.0 & \\
\hline LHS 1678 & 043242.63 & $-3947 \quad 12.3$ & $V$ & $11 \mathrm{~s}$ & 111 & 2003.95-2016.05 & 12.09 & 8 & $49.90 \pm 1.14$ & $1.67 \pm 0.14$ & $51.57 \pm 1.15$ & $1001.0 \pm 0.3$ & $166.8 \pm 0.03$ & 92.0 & $!$ \\
\hline LEHPM 1-3861 & $\begin{array}{llll}05 & 00 & 15.78\end{array}$ & -540627.5 & $I$ & $6 \mathrm{~s}$ & 42 & 2005.90-2011.00 & 5.08 & 10 & $15.58 \pm 1.54$ & $0.63 \pm 0.04$ & $16.21 \pm 1.54$ & $1057.7 \pm 1.1$ & $169.2 \pm 0.10$ & 309.3 & \\
\hline LSR $0609+2319$ & 060952.43 & +231912.8 & $I$ & $6 c$ & 58 & 2006.05-2011.00 & 4.95 & 11 & $20.88 \pm 1.39$ & $1.68 \pm 0.21$ & $22.56 \pm 1.41$ & $1109.5 \pm 0.8$ & $131.3 \pm 0.08$ & 233.1 & \\
\hline SCR 0701-0655 & $\begin{array}{lll}07 & 01 & 17.79\end{array}$ & -065549.4 & $I$ & $4 \mathrm{~s}$ & 49 & 2009.08-2011.20 & 3.12 & 9 & $4.68 \pm 0.98$ & $1.58 \pm 0.14$ & $6.26 \pm 0.99$ & $583.3 \pm 0.8$ & $185.0 \pm 0.12$ & 441.6 & \\
\hline SCR 0708-4709 & 070832.04 & -470930.6 & V & $5 \mathrm{~s}$ & 54 & 2007.19-2011.23 & 4.04 & 9 & $11.24 \pm 1.34$ & $0.81 \pm 0.10$ & $12.05 \pm 1.34$ & $402.2 \pm 1.0$ & $114.8 \pm 0.28$ & 158.3 & \\
\hline SCR 0709-4648 & 070937.28 & -464858.8 & $R$ & $4 c$ & 53 & 2008.14-2011.23 & 3.09 & 9 & $13.20 \pm 1.41$ & $1.22 \pm 0.16$ & $14.42 \pm 1.42$ & $391.5 \pm 1.2$ & $9.0 \pm 0.30$ & 128.7 & \\
\hline SCR 0816-7727 & 081635.65 & -772711.7 & V & $3 \mathrm{~s}$ & 36 & 2010.01-2012.19 & 2.17 & 9 & $12.72 \pm 1.69$ & $0.94 \pm 0.09$ & $13.66 \pm 1.69$ & $688.3 \pm 2.2$ & $324.8 \pm 0.37$ & 238.8 & \\
\hline LHS 2096 & 090308.05 & +084243.8 & $R$ & $4 \mathrm{~s}$ & 51 & 2010.01-2013.39 & 3. & 7 & $16.46 \pm 1.78$ & $0.98 \pm 0.14$ & $17.44 \pm 1.79$ & $549.1 \pm 1.4$ & $250.1 \pm 0.27$ & 49.3 & \\
\hline LHS 2099 & $0905 \quad 28.29$ & -220156.4 & $I$ & $5 \mathrm{~s}$ & 48 & 2006.21-2013.38 & 7.1 & 11 & $18.05 \pm 1.07$ & $0.55 \pm 0.10$ & $18.60 \pm 1.07$ & $622.8 \pm 0.8$ & $173.3 \pm 0.11$ & 58.7 & $!$ \\
\hline LHS 2100 & 090528.29 & -220156.4 & $I$ & $5 \mathrm{~s}$ & 48 & 2006.21-2013.38 & 7.1 & 11 & $21.33 \pm 1.11$ & $0.55 \pm 0.10$ & $21.88 \pm 1.11$ & $624.0 \pm 0.8$ & $173.5 \pm 0.11$ & 35.2 & $!$ \\
\hline LHS 2140 & 092531.09 & +00 1817.6 & $I$ & $6 c$ & 71 & 2008.12-2013.12 & 5.00 & 12 & $16.34 \pm 1.10$ & $0.67 \pm 0.04$ & $17.01 \pm 1.10$ & $577.8 \pm 0.6$ & $186.2 \pm 0.10$ & 61.0 & $!$ \\
\hline LHS 2299 & 104244.78 & -215420.4 & $I$ & $4 c$ & 41 & $2010.16-2013.26$ & 3.10 & 8 & $10.93 \pm 1.40$ & $0.93 \pm 0.12$ & $11.86 \pm 1.41$ & $715.4 \pm 1.1$ & $234.2 \pm 0.17$ & 85.8 & \\
\hline SCR $1227-4541$ & 122746.83 & $\begin{array}{llll}-45 & 41 & 16.9\end{array}$ & $I$ & $4 \mathrm{~s}$ & 39 & 2008.07-2011.24 & 3.17 & 9 & $13.37 \pm 1.47$ & $1.69 \pm 0.11$ & $15.06 \pm 1.47$ & $1286.7 \pm 0.8$ & $282.5 \pm 0.06$ & 404.9 & \\
\hline SSS $1358-3938$ & 135805.40 & -393755.2 & $R$ & $7 \mathrm{c}$ & 118 & 2010.16-2016.19 & 6.03 & 9 & $87.06 \pm 0.78$ & $1.60 \pm 0.17$ & $88.66 \pm 0.80$ & $1959.2 \pm 0.5$ & $117.2 \pm 0.03$ & 104.7 & $!$ \\
\hline LHS 2904 & 142224.92 & -071713.9 & V & $4 \mathrm{~s}$ & 58 & 2009.32-2012.57 & 3.25 & 9 & $18.61 \pm 2.54$ & $1.49 \pm 0.60$ & $20.10 \pm 2.61$ & $651.5 \pm 2.8$ & $247.8 \pm 0.47$ & 153.6 & \\
\hline SCR $1433-3847$ & 143303.33 & -384659.6 & $I$ & $4 \mathrm{c}$ & 47 & 2008.15-2011.20 & 3.13 & 9 & $7.17 \pm 1.00$ & $0.73 \pm 0.09$ & $7.90 \pm 1.00$ & $471.5 \pm 0.8$ & $260.6 \pm 0.17$ & 282.8 & \\
\hline LHS 382 & 145041.22 & -165630.8 & $I$ & $8 \mathrm{~s}$ & 86 & 2001.21-2011.49 & 10.29 & 7 & $20.69 \pm 0.77$ & $0.77 \pm 0.09$ & $21.46 \pm 0.78$ & $1436.0 \pm 0.2$ & $243.8 \pm 0.02$ & 317.1 & \\
\hline SCR 1455-3914 & 145551.56 & $\begin{array}{lll}-39 & 14 & 33.2\end{array}$ & $I$ & $4 \mathrm{c}$ & 51 & 2010.17-2013.39 & 3.22 & 8 & $13.79 \pm 0.96$ & $0.79 \pm 0.07$ & $14.58 \pm 0.96$ & $810.8 \pm 1.1$ & $266.0 \pm 0.12$ & 263.5 & \\
\hline 2MA $1506+1321$ & 150654.35 & +132106.1 & $I$ & $7 \mathrm{c}$ & 53 & 2010.39-2016.21 & 5.82 & 10 & $86.48 \pm 1.58$ & $0.60 \pm 0.04$ & $87.08 \pm 1.58$ & $1063.0 \pm 0.8$ & $270.0 \pm 0.06$ & 58.3 & $!$ \\
\hline SIP $1540-2613$ & 154029.61 & -261343.0 & $I$ & $3 \mathrm{c}$ & 46 & 2010.39-2012.58 & 2.19 & 11 & $66.24 \pm 1.12$ & $0.68 \pm 0.12$ & $66.92 \pm 1.13$ & $1604.4 \pm 1.4$ & $225.5 \pm 0.10$ & 113.6 & \\
\hline SCR $1740-5646$ & 174046.95 & -564658.1 & $I$ & $5 \mathrm{~s}$ & 53 & 2008.31-2012.26 & 3.95 & 9 & $13.71 \pm 1.12$ & $1.13 \pm 0.08$ & $14.84 \pm 1.12$ & $447.2 \pm 0.9$ & $229.1 \pm 0.23$ & 142.9 & \\
\hline SCR $1756-5927$ & 175627.98 & $\begin{array}{llll}-59 & 27 & 18.2\end{array}$ & $I$ & $4 \mathrm{~s}$ & 38 & 2008.40-2011.70 & 3.33 & 8 & $7.21 \pm 1.38$ & $0.75 \pm 0.04$ & $7.96 \pm 1.38$ & $539.1 \pm 1.0$ & $210.8 \pm 0.22$ & 321.2 & \\
\hline SCR 1809-6154B & 180902.62 & -615414.6 & $I$ & $5 \mathrm{~s}$ & 55 & $2010.58-2015.39$ & 4.81 & 13 & $5.73 \pm 1.64$ & $0.49 \pm 0.05$ & $6.22 \pm 1.64$ & $184.3 \pm 1.2$ & $254.4 \pm 0.64$ & 140.4 & $!$ \\
\hline SCR 1809-6154A & 180905.35 & $-6154 \quad 14.5$ & $I$ & $5 \mathrm{~s}$ & 27 & $2010.58-2015.39$ & 4.81 & 13 & $3.38 \pm 1.64$ & $0.49 \pm 0.05$ & $3.87 \pm 1.64$ & $182.1 \pm 1.2$ & $254.3 \pm 0.65$ & 222.9 & $!$ \\
\hline G $182-41 \mathrm{AB}$ & 180926.55 & +275523.3 & $R$ & $4 \mathrm{c}$ & 52 & 2007.44-2010.65 & 3.22 & 10 & $8.85 \pm 2.33$ & $1.11 \pm 0.13$ & $9.96 \pm 2.33$ & $278.0 \pm 2.1$ & $241.5 \pm 0.85$ & 132.3 & $!$ \\
\hline WIS 1912-3615 & 191239.24 & -361456.6 & V & $5 \mathrm{~s}$ & 56 & 2011.50-2015.41 & 3.91 & 9 & $86.22 \pm 1.43$ & $1.02 \pm 0.17$ & $87.24 \pm 1.44$ & $2090.5 \pm 1.0$ & $158.2 \pm 0.05$ & 113.6 & \\
\hline SCR 1913-1001 & 191324.63 & -100146.5 & $I$ & $8 \mathrm{~s}$ & 56 & 2008.70-2015.54 & 6.85 & 8 & $7.90 \pm 0.93$ & $2.60 \pm 0.21$ & $10.50 \pm 0.95$ & $566.3 \pm 0.4$ & $211.8 \pm 0.07$ & 255.6 & \\
\hline USN $2101+0307 \mathrm{AB}$ & 210104.80 & +030704.7 & $I$ & $10 \mathrm{~s}$ & 82 & 2006.79-2015.82 & 9.04 & 8 & $55.54 \pm 1.71$ & $0.89 \pm 0.10$ & $56.43 \pm 1.71$ & $1008.0 \pm 0.6$ & $91.6 \pm 0.05$ & 84.7 & $!$ \\
\hline SCR 2101-5437 & 210145.67 & -543732.0 & $I$ & $4 \mathrm{~s}$ & 37 & 2008.50-2011.80 & 3.13 & 10 & $9.70 \pm 1.38$ & $0.69 \pm 0.03$ & $10.39 \pm 1.38$ & $711.2 \pm 1.1$ & $243.5 \pm 0.16$ & 324.5 & \\
\hline SCR 2204-3347 & 220402.30 & -334738.9 & $I$ & $6 \mathrm{~s}$ & 49 & 2005.70-2010.75 & 5.03 & 9 & $14.25 \pm 1.36$ & $1.51 \pm 0.14$ & $15.76 \pm 1.37$ & $977.0 \pm 0.7$ & $152.4 \pm 0.08$ & 293.9 & \\
\hline LEHPM 1-4592 & $2221 \quad 11.35$ & -195814.8 & $I$ & $8 \mathrm{c}$ & 60 & 2006.43-2015.56 & 9.13 & 9 & $16.51 \pm 1.11$ & $0.33 \pm 0.05$ & $16.84 \pm 1.11$ & $1059.8 \pm 0.4$ & $122.1 \pm 0.04$ & 298.4 & \\
\hline LHS 3841AB & 223959.41 & -361555.7 & $I$ & $5 \mathrm{~s}$ & 60 & $2008.70-2012.88$ & 4.18 & 7 & $11.73 \pm 1.47$ & $0.40 \pm 0.03$ & $12.13 \pm 1.47$ & $900.2 \pm 1.0$ & $170.4 \pm 0.10$ & 351.9 & $!$ \\
\hline LHS 539 & 231551.61 & -373330.6 & $R$ & $4 \mathrm{~s}$ & 57 & 2000.87-2003.77 & 2.89 & 8 & $46.53 \pm 1.00$ & $0.92 \pm 0.07$ & $47.45 \pm 1.00$ & $1309.9 \pm 1.5$ & $77.7 \pm 0.11$ & 130.9 & \\
\hline
\end{tabular}




\begin{tabular}{|c|c|c|c|c|c|c|c|c|c|c|c|c|c|c|c|}
\hline Name & R.A. & 00.0) & Filt & Nsea & Nfrm & Coverage & Years & Nref & $\begin{array}{c}\pi(\mathrm{rel}) \\
(\mathrm{mas}) \\
(9)\end{array}$ & $\begin{array}{c}\pi \text { (corr) } \\
(\mathrm{mas}) \\
(10)\end{array}$ & $\begin{array}{c}\pi(\mathrm{abs}) \\
(\mathrm{mas}) \\
(11)\end{array}$ & $\begin{array}{c}\mu \\
\left(\operatorname{mas~yr}^{-1}\right) \\
(12)\end{array}$ & $\begin{array}{l}\text { P.A. } \\
(\mathrm{deg}) \\
(13)\end{array}$ & $\begin{array}{c}V_{\tan } \\
\left(\mathrm{km} \mathrm{s}^{-1}\right) \\
(14)\end{array}$ & Note \\
\hline \multicolumn{16}{|c|}{ Revised Parallaxes } \\
\hline LHS 178 & $03 \quad 4229.45$ & +123133.7 & V & $4 \mathrm{~s}$ & 44 & 2009.93-2012.95 & 3.02 & 8 & $38.37 \pm 2.48$ & $1.82 \pm 0.27$ & $40.19 \pm 2.49$ & $1571.8 \pm 2.2$ & $153.4 \pm 0.15$ & 185.4 & $!$ \\
\hline G 99-48AB & 055905.98 & +041038.7 & $I$ & $5 c$ & 55 & $2007.81-2012.88$ & 5.07 & 9 & $6.18 \pm 1.83$ & $1.88 \pm 0.30$ & $8.06 \pm 1.85$ & $351.4 \pm 1.5$ & $131.6 \pm 0.49$ & 206.5 & ! \\
\hline LHS 272 & 094346.16 & -174706.2 & V & $9 \mathrm{~s}$ & 93 & 2001.15-2016.05 & 14.91 & 10 & $68.26 \pm 1.01$ & $1.14 \pm 0.11$ & $69.40 \pm 1.02$ & $1432.5 \pm 0.2$ & $279.1 \pm 0.01$ & 97.8 & ! \\
\hline WT 248 & 100554.94 & -672131.2 & $I$ & $4 \mathrm{c}$ & 50 & $2000.14-2003.25$ & 3.10 & 11 & $40.52 \pm 2.23$ & $1.12 \pm 0.08$ & $41.64 \pm 2.23$ & $1214.4 \pm 1.8$ & $264.8 \pm 0.13$ & 138.2 & ! \\
\hline G $10-3$ & 111002.64 & -024726.4 & V & $7 \mathrm{~s}$ & 63 & $2010.17-2016.19$ & 6.02 & 7 & $6.63 \pm 1.85$ & $0.85 \pm 0.17$ & $7.48 \pm 1.86$ & $493.7 \pm 0.8$ & $157.6 \pm 0.18$ & 313.1 & $!$ \\
\hline LHS 334 & 123415.78 & +203705.7 & $I$ & $7 \mathrm{~s}$ & 47 & 2003.24-2011.11 & 7.87 & 6 & $16.78 \pm 1.99$ & $0.70 \pm 0.06$ & $17.48 \pm 1.99$ & $1333.8 \pm 0.7$ & $165.6 \pm 0.05$ & 361.8 & $!$ \\
\hline LHS 2852 & 140246.66 & -243149.6 & $R$ & $4 \mathrm{c}$ & 60 & 2008.20-2011.42 & 3.22 & 8 & $56.43 \pm 1.83$ & $1.56 \pm 0.44$ & $57.99 \pm 1.88$ & $512.8 \pm 1.6$ & $317.0 \pm 0.35$ & 41.9 & ! \\
\hline SSS $1444-2019$ & 144420.33 & -201925.5 & $I$ & $6 \mathrm{~s}$ & 50 & $2010.20-2016.47$ & 6.27 & 8 & $59.83 \pm 1.62$ & $0.35 \pm 0.03$ & $60.18 \pm 1.62$ & $3495.1 \pm 1.1$ & $235.9 \pm 0.04$ & 275.3 & ! \\
\hline LHS 385 & 145535.83 & -153344.0 & V & $5 \mathrm{~s}$ & 49 & $2003.24-2012.58$ & 9.34 & 9 & $23.62 \pm 1.51$ & $0.81 \pm 0.11$ & $24.43 \pm 1.51$ & $1727.8 \pm 0.9$ & $210.5 \pm 0.06$ & 335.2 & ! \\
\hline LHS 401 & 153939.06 & -550910.0 & V & $3 \mathrm{c}$ & 59 & $2010.16-2012.58$ & 2.42 & 10 & $34.40 \pm 1.42$ & $4.27 \pm 0.90$ & $38.67 \pm 1.68$ & $1122.1 \pm 1.8$ & $188.2 \pm 0.15$ & 137.5 & $!$ \\
\hline LSR $1610-0040 \mathrm{AB}$ & 161028.96 & -004054.0 & $I$ & $11 \mathrm{~s}$ & 140 & $2006.21-2016.19$ & 9.98 & 13 & $31.02 \pm 0.46$ & $1.24 \pm 0.14$ & $32.26 \pm 0.48$ & $1448.7 \pm 0.2$ & $213.5 \pm 0.01$ & 212.8 & ! \\
\hline LHS 440AB & 171825.58 & -432637.6 & $R$ & $12 \mathrm{~s}$ & 177 & $2000.58-2015.56$ & 14.99 & 10 & $37.68 \pm 0.87$ & $1.88 \pm 0.54$ & $39.56 \pm 1.02$ & $1080.2 \pm 0.2$ & $233.1 \pm 0.02$ & 129.4 & ! \\
\hline LHS 456 & 175058.99 & -563606.8 & V & $5 \mathrm{~s}$ & 49 & $1999.50-2010.50$ & 11.10 & 9 & $39.29 \pm 1.43$ & $0.58 \pm 0.11$ & $39.87 \pm 1.43$ & $1256.9 \pm 0.5$ & $238.1 \pm 0.04$ & 149.4 & ! \\
\hline LHS 72 & 234313.65 & -240952.1 & V & $3 c$ & 64 & $2010.50-2012.87$ & 2.37 & 7 & $33.23 \pm 1.62$ & $1.97 \pm 0.19$ & $35.20 \pm 1.63$ & $2558.2 \pm 1.9$ & $150.2 \pm 0.08$ & 344.5 & ! \\
\hline LHS 73 & 234313.65 & -240952.1 & V & $3 c$ & 64 & $2010.50-2012.87$ & 2.37 & 7 & $30.76 \pm 1.43$ & $1.97 \pm 0.19$ & $32.73 \pm 1.44$ & $2554.6 \pm 1.6$ & $150.1 \pm 0.07$ & 370.0 & $!$ \\
\hline
\end{tabular}

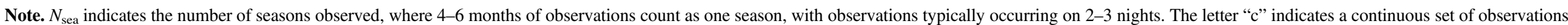

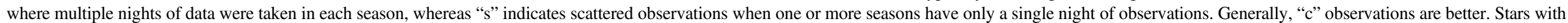
exclamation marks in the Notes column are discussed in Section 4.

(This table is available in machine-readable form.) 
Table 2

Photometry and Spectroscopy Results

\begin{tabular}{|c|c|c|c|c|c|c|c|c|c|c|c|c|}
\hline Name1 & $\begin{array}{l}\text { Name2 } \\
\text { (2) }\end{array}$ & $\begin{array}{c}V \\
\text { mag } \\
(3)\end{array}$ & $\begin{array}{c}R \\
\text { mag } \\
(4)\end{array}$ & $\begin{array}{c}I \\
\mathrm{mag} \\
(5)\end{array}$ & $\#$ & $\begin{array}{l}\pi \\
\text { filter } \\
\text { (7) }\end{array}$ & $\begin{array}{c}\sigma \\
\mathrm{mag} \\
(8)\end{array}$ & $\begin{array}{c}J \\
\operatorname{mag} \\
(9)\end{array}$ & $\begin{array}{c}H \\
\mathrm{mag} \\
(10)\end{array}$ & $\begin{array}{c}K_{s} \\
\mathrm{mag} \\
(11)\end{array}$ & $\begin{array}{l}\text { Spect. } \\
\text { (12) }\end{array}$ & $\begin{array}{c}\text { Reference } \\
\text { (13) }\end{array}$ \\
\hline LHS 1048 & G $267-58$ & 14.53 & 13.47 & 12.09 & 2 & $I$ & 0.0094 & $10.80 \pm 0.02$ & $10.26 \pm 0.02$ & $10.06 \pm 0.02$ & M4 & 1 \\
\hline LHS 127 & G $268-77$ & 15.79 & 14.77 & 13.61 & 2 & $I$ & 0.0091 & $12.46 \pm 0.02$ & $11.92 \pm 0.02$ & $11.73 \pm 0.02$ & M2.0VI & 8 \\
\hline LEHPM 1-1628 & & 17.11 & 16.16 & 15.23 & 2 & $I$ & 0.0197 & $14.14 \pm 0.03$ & $13.71 \pm 0.03$ & $13.46 \pm 0.04$ & M1.0VI & 8 \\
\hline LHS 1257 & LSPM J0131+1001 & 16.37 & 15.26 & 13.82 & 2 & $I$ & 0.0103 & $12.40 \pm 0.02$ & $11.93 \pm 0.02$ & $11.68 \pm 0.02$ & VI & 16 \\
\hline LHS 150 & GJ 85 & 11.49 & 10.49 & 9.31 & 3 & V & 0.0092 & $8.13 \pm 0.02$ & $7.61 \pm 0.03$ & $7.36 \pm 0.02$ & $\mathrm{M} 1.5 \mathrm{~V}$ & 6 \\
\hline LHS 1490 & LP 994-33 & 15.87 & 14.35 & 12.44 & 3 & $I$ & 0.0104 & $10.71 \pm 0.02$ & $10.18 \pm 0.03$ & $9.88 \pm 0.02$ & M5.0VI & 8 \\
\hline LHS 1678 & LP $375-2$ & 12.48 & 11.46 & 10.26 & 3 & $V$ & 0.0064 & $9.02 \pm 0.03$ & $8.50 \pm 0.05$ & $8.26 \pm 0.03$ & $\mathrm{M} 2.0 \mathrm{~V}$ & 12 \\
\hline LEHPM 1-3861 & SSSPM J0500-5406 & 18.48 & 17.24 & 15.77 & 2 & $I$ & 0.0107 & $14.44 \pm 0.03$ & $14.12 \pm 0.05$ & $13.97 \pm 0.06$ & M4.0VI & 8 \\
\hline LSR 0609+2319 & LSPM J0609+2319 & 17.65 & 16.33 & 14.65 & 2 & $I$ & 0.0078 & $13.16 \pm 0.02$ & $12.64 \pm 0.02$ & $12.41 \pm 0.02$ & sdM5.0 & 10 \\
\hline SCR 0701-0655 & & 16.55 & 15.61 & 14.75 & 2 & $I$ & 0.0088 & $13.73 \pm 0.02$ & $13.19 \pm 0.02$ & $13.00 \pm 0.03$ & M1.0VI & 8 \\
\hline SCR $0708-4709$ & & 13.81 & 13.05 & 12.37 & 3 & V & 0.0078 & $11.44 \pm 0.02$ & $10.90 \pm 0.02$ & $10.76 \pm 0.03$ & K7.0VI & 8 \\
\hline SCR 0709-4648 & PM J07096-4648 & 14.91 & 14.02 & 13.22 & 2 & $R$ & 0.0088 & $12.20 \pm 0.03$ & $11.70 \pm 0.03$ & $11.49 \pm 0.03$ & $\mathrm{M} 0.5 \mathrm{VI}$ & 8 \\
\hline SCR 0816-7727 & & 15.30 & 14.40 & 13.58 & 3 & V & 0.0070 & $12.62 \pm 0.03$ & $12.07 \pm 0.02$ & $11.87 \pm 0.02$ & VI & 16 \\
\hline LHS 2096 & LP $486-42$ & 17.81 & 16.64 & 15.29 & 3 & $R$ & 0.0093 & $13.99 \pm 0.02$ & $13.58 \pm 0.03$ & $13.41 \pm 0.04$ & esdM5.5 & 11 \\
\hline LHS 2099 & LP $845-16$ & 15.83 & 14.84 & 13.79 & 3 & $I$ & 0.0088 & $12.64 \pm 0.03$ & $12.15 \pm 0.04$ & $11.94 \pm 0.03$ & esdM2.0 & 11 \\
\hline LHS 2100 & LP $845-17$ & 19.16 & 17.73 & 15.80 & 3 & $I$ & 0.0170 & $14.27 \pm 0.03$ & $13.87 \pm 0.04$ & $13.63 \pm 0.05$ & esdM2.0 & 11 \\
\hline LHS 2140 & G $46-40$ & 15.05 & 14.13 & 13.24 & 4 & $I$ & 0.0099 & $12.17 \pm 0.02$ & $11.63 \pm 0.02$ & $11.44 \pm 0.03$ & VI & 3 \\
\hline LHS 2139 & & 19.56 & 18.93 & 18.34 & 4 & $I$ & $\ldots$ & $\ldots$ & $\ldots$ & $\ldots$ & WD & 3 \\
\hline LHS 2299 & LP 790-36 & 16.95 & 15.90 & 14.69 & 2 & $I$ & 0.0077 & $13.52 \pm 0.02$ & $12.98 \pm 0.02$ & $12.74 \pm 0.03$ & sdM3.0 & 11 \\
\hline SCR $1227-4541$ & PM J12277-4541 & 15.23 & 14.40 & 13.69 & 2 & $I$ & 0.0079 & $12.75 \pm 0.03$ & $12.39 \pm 0.03$ & $12.27 \pm 0.03$ & VI & 16 \\
\hline SSS 1358-3938 & & 14.04 & 12.80 & 11.20 & 2 & $R$ & 0.0110 & $9.72 \pm 0.02$ & $9.23 \pm 0.02$ & $8.95 \pm 0.02$ & VI & 16 \\
\hline LHS 2904 & G $124-29$ & 12.40 & 11.60 & 10.87 & 3 & V & 0.0074 & $9.93 \pm 0.02$ & $9.31 \pm 0.02$ & $9.15 \pm 0.02$ & VI & 16 \\
\hline SCR $1433-3847$ & PM J14330-3846 & 17.23 & 16.29 & 15.41 & 3 & $I$ & 0.0086 & $14.37 \pm 0.04$ & $13.78 \pm 0.05$ & $13.59 \pm 0.05$ & $\mathrm{M} 0.5 \mathrm{VI}$ & 8 \\
\hline LHS 382 & LP $801-16$ & 15.73 & 14.61 & 13.16 & 2 & $I$ & 0.0079 & $11.85 \pm 0.02$ & $11.38 \pm 0.03$ & $11.11 \pm 0.02$ & M1.5 & 15 \\
\hline SCR $1455-3914$ & PM J14558-3914 & 15.43 & 14.50 & 13.58 & 2 & $I$ & 0.0087 & $12.50 \pm 0.02$ & $11.98 \pm 0.02$ & $11.79 \pm 0.02$ & M1.0VI & 8 \\
\hline 2MA $1506+1321$ & & $\ldots$ & 19.30 & 16.93 & 2 & $I$ & 0.0286 & $13.37 \pm 0.02$ & $12.38 \pm 0.02$ & $11.74 \pm 0.02$ & L3.0 & 4 \\
\hline SIP $1540-2613$ & & 19.21 & 16.57 & 14.11 & 1 & $I$ & 0.0089 & $11.65 \pm 0.03$ & $11.14 \pm 0.03$ & $10.73 \pm 0.02$ & V & 16 \\
\hline SCR $1740-5646$ & & 17.03 & 16.01 & 14.95 & 3 & $I$ & 0.0099 & $13.83 \pm 0.03$ & $13.33 \pm 0.03$ & $13.20 \pm 0.04$ & M3.0VI & 8 \\
\hline SCR 1756-5927 & & 16.30 & 15.38 & 14.49 & 2 & $I$ & 0.0092 & $13.44 \pm 0.03$ & $12.89 \pm 0.03$ & $12.69 \pm 0.03$ & M1.0VI & 8 \\
\hline SCR 1809-6154A & & 15.66 & 14.79 & 13.92 & 2 & $I$ & $\ldots$ & $12.84 \pm 0.03$ & $12.32 \pm 0.03$ & $12.09 \pm 0.02$ & VI & 16 \\
\hline SCR 1809-6154B & & 16.11 & 15.19 & 14.21 & 2 & $I$ & 0.0094 & $13.14 \pm 0.03$ & $12.57 \pm 0.03$ & $12.43 \pm 0.02$ & VI & 16 \\
\hline G $182-41 \mathrm{AB}$ & LP $334-10$ & $12.62 \mathrm{~J}$ & $12.06 \mathrm{~J}$ & $11.53 \mathrm{~J}$ & 3 & $R$ & 0.0088 & $10.74 \pm 0.02 \mathrm{~J}$ & $10.26 \pm 0.03 \mathrm{~J}$ & $10.15 \pm 0.02$ & VI & 16 \\
\hline WIS 1912-3615 & & 13.91 & 12.64 & 11.00 & 3 & V & 0.0095 & $9.52 \pm 0.02$ & $9.01 \pm 0.06$ & $8.77 \pm 0.02$ & mid-M & 5 \\
\hline SCR 1913-1001 & & 15.60 & 14.66 & 13.80 & 3 & $I$ & 0.0119 & $12.71 \pm 0.03$ & $12.16 \pm 0.03$ & $11.93 \pm 0.03$ & VI & 16 \\
\hline USN $2101+0307 \mathrm{AB}$ & & $18.67 \mathrm{~J}$ & $16.64 \mathrm{~J}$ & $14.32 \mathrm{~J}$ & 3 & $I$ & 0.0090 & $11.70 \pm 0.02 \mathrm{~J}$ & $10.96 \pm 0.02 \mathrm{~J}$ & $10.57 \pm 0.02 \mathrm{~J}$ & $\mathrm{~V}$ & 16 \\
\hline SCR 2101-5437 & & 15.77 & 14.84 & 13.86 & 3 & $I$ & 0.0066 & $12.79 \pm 0.03$ & $12.26 \pm 0.02$ & $12.08 \pm 0.03$ & M1.0VI & 8 \\
\hline SCR 2204-3347 & & 15.44 & 14.45 & 13.41 & 3 & $I$ & 0.0074 & $12.32 \pm 0.03$ & $11.81 \pm 0.03$ & $11.60 \pm 0.03$ & $\mathrm{M} 3.0 \mathrm{VI}$ & 8 \\
\hline LEHPM 1-4592 & & 19.96 & 18.16 & 15.98 & 3 & $I$ & 0.0091 & $14.19 \pm 0.03$ & $13.74 \pm 0.04$ & $13.48 \pm 0.04$ & VI & 16 \\
\hline LHS 3841AB & LP 984-76 & $16.87 \mathrm{~J}$ & $15.89 \mathrm{~J}$ & $14.89 \mathrm{~J}$ & 2 & $I$ & 0.0061 & $13.82 \pm 0.02 \mathrm{~J}$ & $13.32 \pm 0.03 \mathrm{~J}$ & $13.20 \pm 0.04 \mathrm{~J}$ & $\mathrm{sdM} 2.5$ & 11 \\
\hline LHS 539 & LP 986-16 & 14.97 & 13.66 & 11.98 & 3 & $R$ & 0.0107 & $10.40 \pm 0.02$ & $9.87 \pm 0.02$ & $9.59 \pm 0.02$ & $\mathrm{~V}$ & 16 \\
\hline LHS 178 & G 79-59 & 12.87 & 11.89 & 10.78 & 2 & $V$ & 0.0076 & $9.60 \pm 0.02$ & $9.11 \pm 0.02$ & $8.88 \pm 0.02$ & sdM1.5 & 3 \\
\hline G $99-48 \mathrm{AB}$ & LTT 17896AB & $11.85 \mathrm{~J}$ & $11.42 \mathrm{~J}$ & $10.97 \mathrm{~J}$ & 3 & $I$ & 0.0086 & $10.38 \pm 0.03 \mathrm{~J}$ & $9.99 \pm 0.02 \mathrm{~J}$ & $9.89 \pm 0.02 \mathrm{~J}$ & VI & 16 \\
\hline LHS 272 & LP 788-27 & 13.16 & 12.10 & 10.87 & 3 & V & 0.0125 & $9.62 \pm 0.02$ & $9.12 \pm 0.02$ & $8.87 \pm 0.02$ & M3.0VI & 9 \\
\hline WT 248 & & 14.52 & 13.40 & 11.95 & 2 & $I$ & 0.0077 & $10.56 \pm 0.02$ & $10.10 \pm 0.02$ & $9.87 \pm 0.02$ & $\mathrm{M} 3.0 \mathrm{~V}$ & 7 \\
\hline G $10-3$ & LHS 2361 & 12.56 & 12.01 & 11.50 & 2 & V & 0.0107 & $10.73 \pm 0.02$ & $10.27 \pm 0.02$ & $10.11 \pm 0.02$ & VI & 8 \\
\hline LHS 334 & LP $377-13$ & 17.99 & 16.75 & 15.13 & 3 & $I$ & 0.0102 & $13.75 \pm 0.03$ & $13.25 \pm 0.04$ & $13.04 \pm 0.03$ & M6.0VI & 8 \\
\hline
\end{tabular}


Table 2

(Continued)

\begin{tabular}{|c|c|c|c|c|c|c|c|c|c|c|c|c|}
\hline Name1 & $\begin{array}{l}\text { Name2 } \\
\text { (2) }\end{array}$ & $\begin{array}{c}V \\
\text { mag } \\
(3)\end{array}$ & $\begin{array}{c}R \\
\text { mag } \\
(4)\end{array}$ & $\begin{array}{c}I \\
\operatorname{mag} \\
(5)\end{array}$ & (6) & $\begin{array}{l}\pi \\
\text { filter } \\
\text { (7) }\end{array}$ & $\begin{array}{c}\sigma \\
\operatorname{mag} \\
(8)\end{array}$ & $\begin{array}{c}J \\
\text { mag } \\
(9)\end{array}$ & $\begin{array}{c}H \\
\mathrm{mag} \\
(10)\end{array}$ & $\begin{array}{c}K_{s} \\
\mathrm{mag} \\
(11)\end{array}$ & $\begin{array}{l}\text { Spect. } \\
\text { (12) }\end{array}$ & $\begin{array}{c}\text { References } \\
\text { (13) }\end{array}$ \\
\hline LHS 2852 & LP $856-36$ & 12.13 & 11.08 & 9.85 & 2 & $R$ & 0.0242 & $8.63 \pm 0.03$ & $8.10 \pm 0.03$ & $7.84 \pm 0.02$ & $\operatorname{sdM} 2.0$ & 3 \\
\hline SSS 1444-2019 & LP $741-20$ & 20.25 & 17.62 & 14.95 & 4 & $I$ & 0.0130 & $12.55 \pm 0.03$ & $12.14 \pm 0.03$ & $11.93 \pm 0.03$ & sdM9 & 14 \\
\hline LHS 385 & LP $741-20$ & 14.61 & 13.67 & 12.78 & 3 & $V$ & 0.0087 & $11.74 \pm 0.03$ & $11.28 \pm 0.02$ & $11.06 \pm 0.02$ & M1.0VI & 8 \\
\hline LHS 401 & L $201-12$ & 12.73 & 11.88 & 11.12 & 2 & $V$ & 0.0077 & $10.15 \pm 0.02$ & $9.60 \pm 0.02$ & $9.41 \pm 0.02$ & M0.5VI & 8 \\
\hline LSR $1610-0040 \mathrm{AB}$ & & $19.09 \mathrm{~J}$ & $17.10 \mathrm{~J}$ & $14.97 \mathrm{~J}$ & 2 & $I$ & 0.0077 & $12.91 \pm 0.02 \mathrm{~J}$ & $12.30 \pm 0.02 \mathrm{~J}$ & $12.02 \pm 0.03 \mathrm{~J}$ & sd?M6pec & 2 \\
\hline LHS $440 \mathrm{AB}$ & L $413-156$ & $12.98 \mathrm{~J}$ & $11.98 \mathrm{~J}$ & $10.87 \mathrm{~J}$ & 3 & $R$ & 0.0113 & $9.70 \pm 0.02 \mathrm{~J}$ & $9.13 \pm 0.02 \mathrm{~J}$ & $8.95 \pm 0.02 \mathrm{~J}$ & M1.0VI & 8 \\
\hline LHS 456 & L $205-83$ & 12.08 & 11.12 & 10.09 & 3 & $V$ & 0.1282 & $8.99 \pm 0.02$ & $8.42 \pm 0.02$ & $8.19 \pm 0.02$ & M2.0 & 15 \\
\hline LHS 72 & G $275-90$ & 12.07 & 11.24 & 10.49 & 3 & $V$ & 0.0087 & $9.61 \pm 0.03$ & $9.04 \pm 0.02$ & $8.82 \pm 0.02$ & VI & 13 \\
\hline LHS 73 & G $275-92$ & 12.77 & 11.90 & 11.10 & 3 & $V$ & 0.0093 & $10.11 \pm 0.02$ & $9.59 \pm 0.02$ & $9.37 \pm 0.02$ & K6.0VI & 8 \\
\hline
\end{tabular}

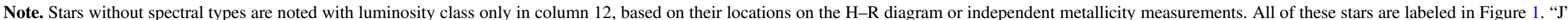
next to a magnitude indicates a combined photometry.

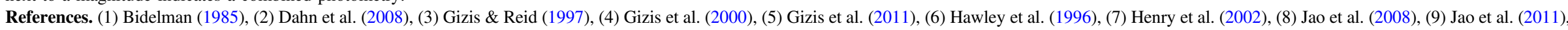
(10) Reid (2003), (11) Reid \& Gizis (2005), (12) Reid et al. (2007), (13) Rodgers \& Eggen (1974), (14) Scholz et al. (2004b), (15) Walker (1983), (16) this work.

(This table is available in machine-readable form.) 


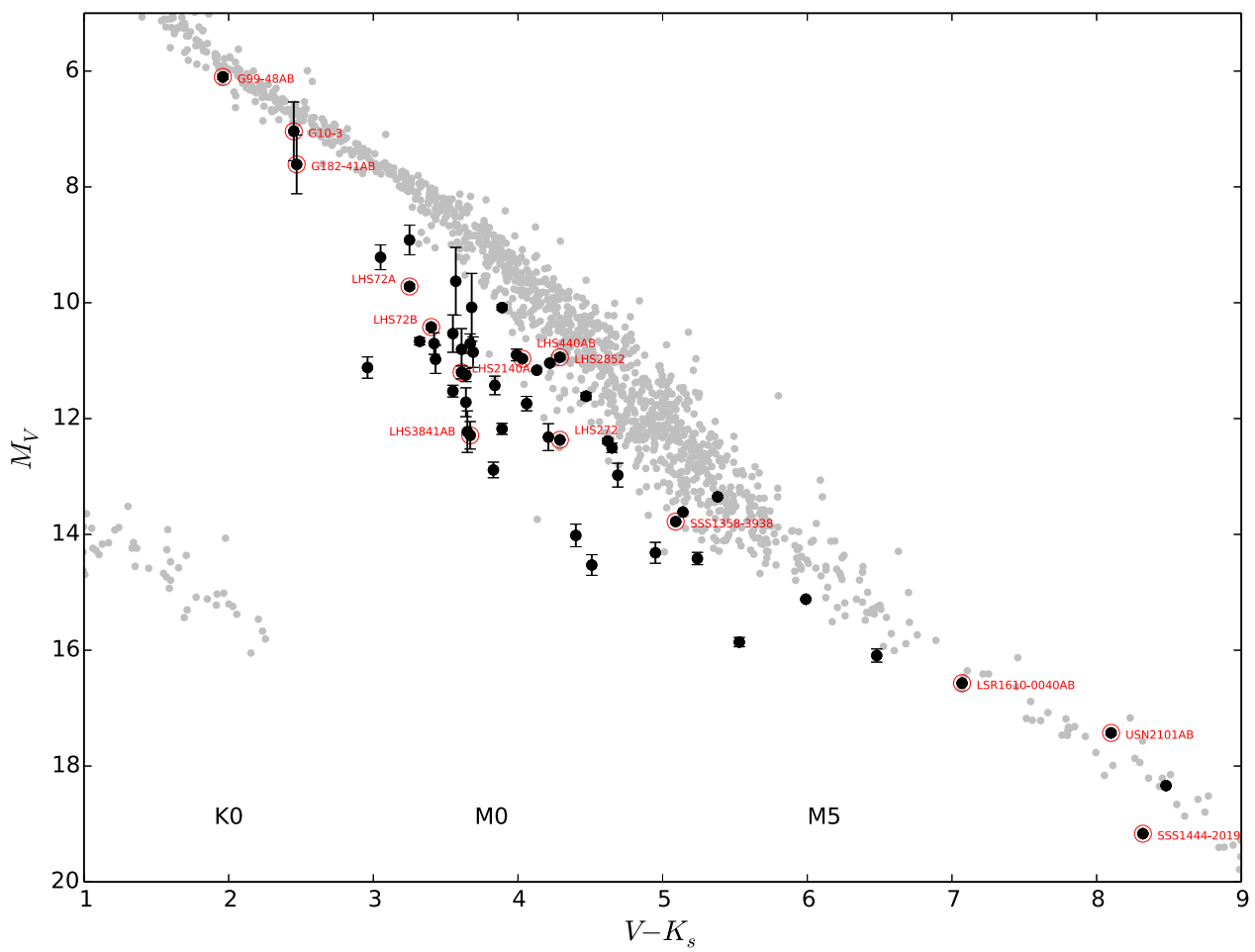

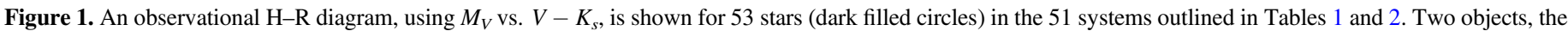

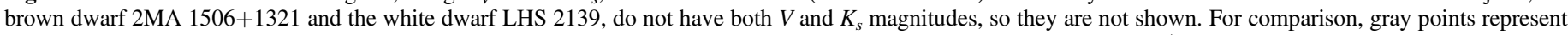

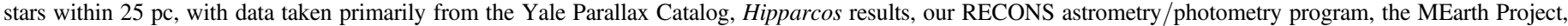

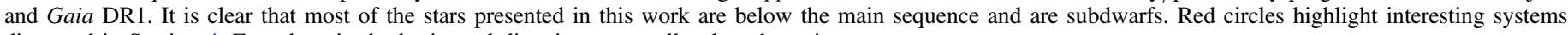
discussed in Section 4. Error bars in the horizontal direction are smaller than the points.

subdwarfs, and three as main sequence stars of class "V." As discussed in Jao et al. (2008), the "sd" prefix often used to classify cool subdwarfs is the same prefix used for hot subdwarfs, even though they are completely different types of stellar objects. The mixed use of "sd" is unique in spectral classification, so we prefer the VI designation. In support of this spectral type moniker, Figures 1, 5, 6, and 7 all clearly show a different luminosity class on the HertzsprungRussell (H-R) diagram for a given $V-K_{s}$ between (at least) 3 and 6 .

\section{Notes on Individual Systems}

Here, we provide additional details of systems worthy of note, listed in order of R.A.

$0342+1231$ (LHS 178). The Yale Parallax Catalog (YPC, van Altena et al. 1995) provides a parallax of $45.1 \pm 12.0$ mas for this star and we find $40.19 \pm 2.49$ mas, resulting in a weighted mean value of $40.39 \pm 2.44$ mas.

0432-3947 (LHS 1678). We detect a possible perturbation with a period of a few years in the astrometric series spanning 12 years, but because it is slight, we have not removed the perturbation to calculate the parallax presented here. Until there is further evidence to support the existence of a currently unseen companion, we consider this is a single star.

$0559+0410(G 99-48 A B)$. Goldberg et al. (2002) found this system to be a double-lined spectroscopic binary, and Soubiran et al. (2010) determined it have $[\mathrm{Fe} / \mathrm{H}]=-1.80$. Our parallax of $8.06 \pm 1.85$ mas is consistent with that provided in Gaia Data Release 1 (hereafter DR1), $7.06 \pm 0.25$ mas.

0905-2201 (LHS 2099/2100). This pair of subdwarfs is separated by 6 ! $^{\prime} 6$ at a position angle of $100^{\circ}$, corresponding to
$326 \mathrm{au}$ at a distance of $49.4 \mathrm{pc}$ for the weighed mean parallax of $20.24 \pm 0.78$ mas. The secondary has $M_{V}=15.69$ and $\left(V-K_{s}\right)=5.53$, placing it well below the main sequence in Figure 1 and making it one of the reddest subdwarfs in the sample.

0925+0018 (LHS 2140 and LHS 2139). Gizis \& Reid (1997) first reported this common proper motion binary to consist of a subdwarf primary (LHS 2140) and a white dwarf secondary (LHS 2139). LHS 2139 is too faint in our images to measure a reliable parallax, so we adopt the parallax of LHS 2140 for both components. This is one of the very few known subdwarf + white dwarf binaries with parallaxes in the solar neighborhood (Monteiro et al. 2006).

0943-1747 (LHS 272). The updated parallax (69.4 \pm 1.02 mas) has a longer time coverage than previously reported in Jao et al. (2011), and supersedes our previous result. This is the fourthnearest known subdwarf system of any spectral type, ranking behind Kapteyn's Star (GJ 191, M type), $\mu$ Cas AB (GJ 53AB, G and $M$ types), and GJ 451 (K type).

1005-6721 (WT 248). Faherty et al. (2012) reported a parallax of $30.6 \pm 4.6$ mas for this object. Our parallax of $41.64 \pm 2.23$ places the system within $25 \mathrm{pc}$, but the weighted mean of $39.54 \pm 2.00$ mas is still slightly less than 40 mas.

1110-0247 (G 10-3). Bidelman (1985) classified this star as a K2 dwarf, but it is undoubtedly a subdwarf, given that Latham et al. (2002) report the star to have $[m / H]=-2.0$. YPC provides a parallax of $28.1 \pm 13.4$ mas and we find $7.48 \pm 1.86$, yielding a weighted mean value of $7.87 \pm$ 1.84 mas.

$1234+2037$ (LHS 334). Our parallax of $17.48 \pm 1.99$ mas is consistent with that of Smart et al. (2010), who reported a 

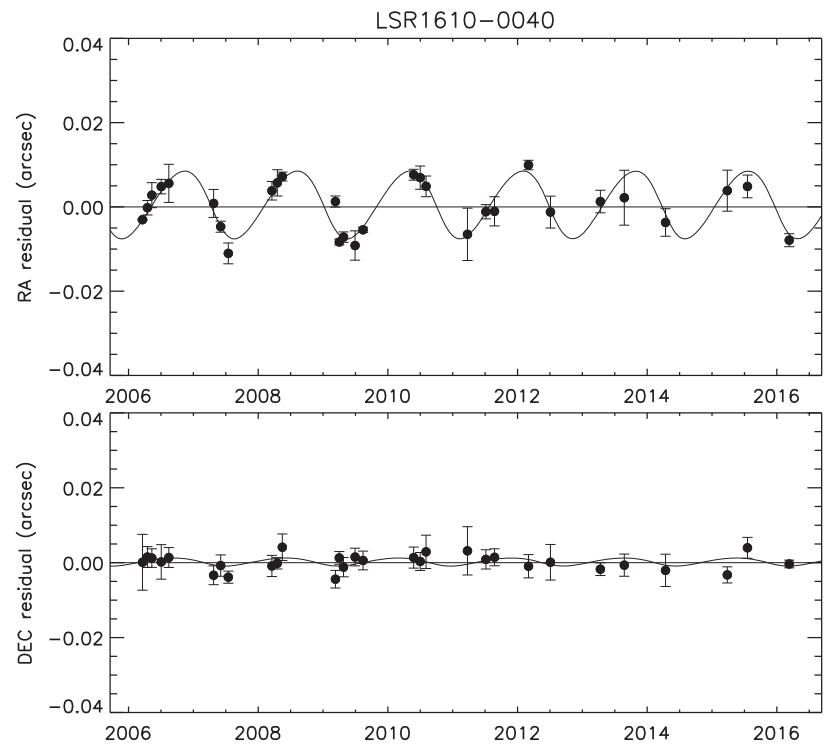

(a)
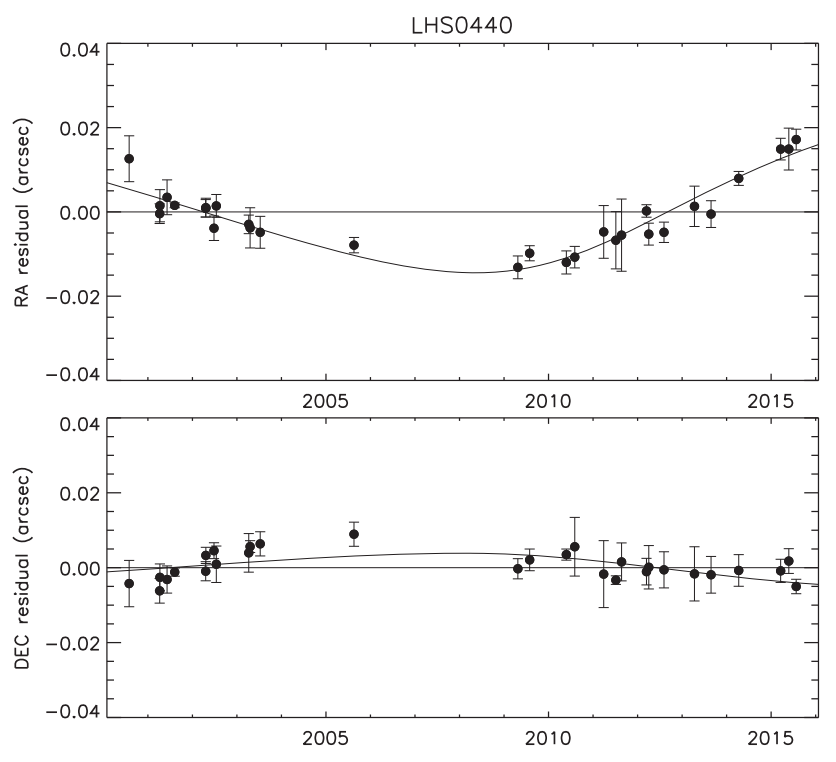

(b)

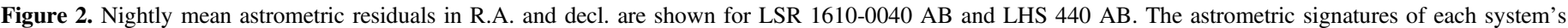

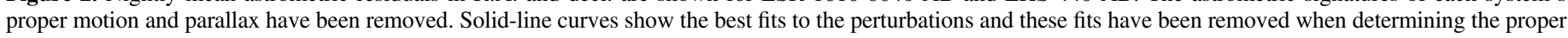
motions and trigonometric parallaxes presented here.

parallax of $22.1 \pm 3.9$ mas. The new weighted mean parallax is $18.43 \pm 1.77$ mas.

1358-3938 (SSS 1358-3938). This star has a proper motion of nearly $2^{\prime \prime} \mathrm{yr}^{-1}$ and we provide the first parallax here, $88.66 \pm 0.80$ mas. The star is located on the edge of the main sequence band in the $\mathrm{H}-\mathrm{R}$ diagram of Figure 1. The photometric distance determined using VRIJHK and the relations of Henry et al. (2004) place this star at $21.2 \mathrm{pc}$, but our parallax puts it at $11.3 \mathrm{pc}$. With no previous spectral type available, this large distance mismatch implies this star is likely a subdwarf and we assign it as "VI." If spectroscopically confirmed, this star would replace LHS 272 as the fourthclosest subdwarf system and the third closest $\mathrm{M}$ type subdwarf.

1402-2431 (LHS 2852). Gizis (1997) identified this star to be a subdwarf, but it is located on the lower edge of the main sequence in the $\mathrm{H}-\mathrm{R}$ diagram of Figure 1. Hakonsen \& Rutledge (2009) found it to have ROSAT X-ray detection of $0.15 \pm 0.03 \mathrm{cnt} \mathrm{s}^{-1}$. In comparison, the known young star AP Col Riedel et al. (2011) has $0.43 \pm 0.05$. In addition, LHS2852 varies by 24 mmag in the $R$ band, the largest variability seen among the 42 subdwarfs studied here. It would be unusual for a subdwarf to be so variable and detected in X-rays, so follow-up spectroscopy is needed to confirm whether or not the star is a subdwarf. YPC provides a parallax of $39.4 \pm 19.9$ mas and we find $57.99 \pm 1.88$ mas, yielding a weighted mean value of $57.83 \pm 1.87$ mas.

1444-2019 (SSS 1444-2019). This high proper motion (nearly 3." $5 \mathrm{yr}^{-1}$ ) star was first detected and classified as an M9 subdwarf by Scholz et al. (2004b). Recently, Kirkpatrick et al. (2016) re-classified it as a L0 subdwarf. Our parallax of $60.18 \pm 1.62$ mas is consistent with the parallaxes reported by Schilbach et al. (2009) (61.67 \pm 2.12 mas) and Faherty et al. (2012) (61.2 \pm 5.1 mas). Together, the three values result in a weighted mean parallax of $60.76 \pm 1.25$ mas.

1455-1533 (LHS 385). YPC provides a parallax of $20.4 \pm 5.8$ mas and we find $24.43 \pm 1.51$ mas, resulting in a weighted mean value of $24.17 \pm 1.46$ mas.
1506+1321 (2MA1506+1321). Gizis et al. (2000) reported this object to be an L3 dwarf, implying that it is a brown dwarf because it is later than the L2 type found by Dieterich et al. (2014) at the stellar/substellar boundary. It is too faint for $V$ band photometry at the $0.9 \mathrm{~m}$, so no DCR correction was made for this field; however, because this field was observed in the $I$ band, any DCR correction is minimal. Gagné et al. (2014) flagged this object with a $100 \%$ probability to be a young field object. The object shows three signs of youth: (1) a triangularshaped H-band continuum, (2) redder-than-normal colors for its assigned spectral type, and (3) signs of low gravity from atmospheric model fitting. With a relatively slow tangential velocity of $58.3 \mathrm{~km} \mathrm{~s}^{-1}$, it does not have the typical high velocity of an old subdwarf, so it is likely a young brown dwarf for which we provide the first parallax of $87.08 \pm 1.58$ mas.

1539-5509 (LHS 401). YPC provides a parallax of $38.4 \pm 9.6$ mas and we find $38.67 \pm 1.68$ mas, resulting in a weighted mean value of $38.66 \pm 1.65$ mas.

1610-0040 (LSR1610-0040 AB) is an important subdwarf system, given that it promises to yield accurate masses for a pair of very cool subdwarfs. This system was first detected and classified as an early-type L subdwarf by Lépine et al. (2003a). Cushing \& Vacca (2006) later showed the system to have a peculiar spectrum with an ambiguous assignment of dwarf or subdwarf type. Dahn et al. (2008) reported the first trigonometric parallax of $31.02 \pm 0.26$ mas, detected a clear photocentric orbit with a semimajor axis of 8.91 mas, and classified it as type "sd?M6pec." Recently, Koren et al. (2016) estimated the masses of the primary and unseen secondary using updated astrometry from Dahn et al. (2008) and radial velocity data from Blake et al. (2010) and found masses of 0.09-0.10 $M_{\odot}$ and $0.06-0.075 M_{\odot}$. Our data set of 9.98 years also detects a large astrometric perturbation (see Figure 2(a)) with a period of 634.8 days, nearly identical to the period of 633 days found by Koren et al. (2016), even though their timespan is 10.2 years. We measure a photocentric semimajor axis of $8.25 \pm 0.63$ mas, but Koren et al. (2016) has $9.89 \pm 0.25$ mas. Although 


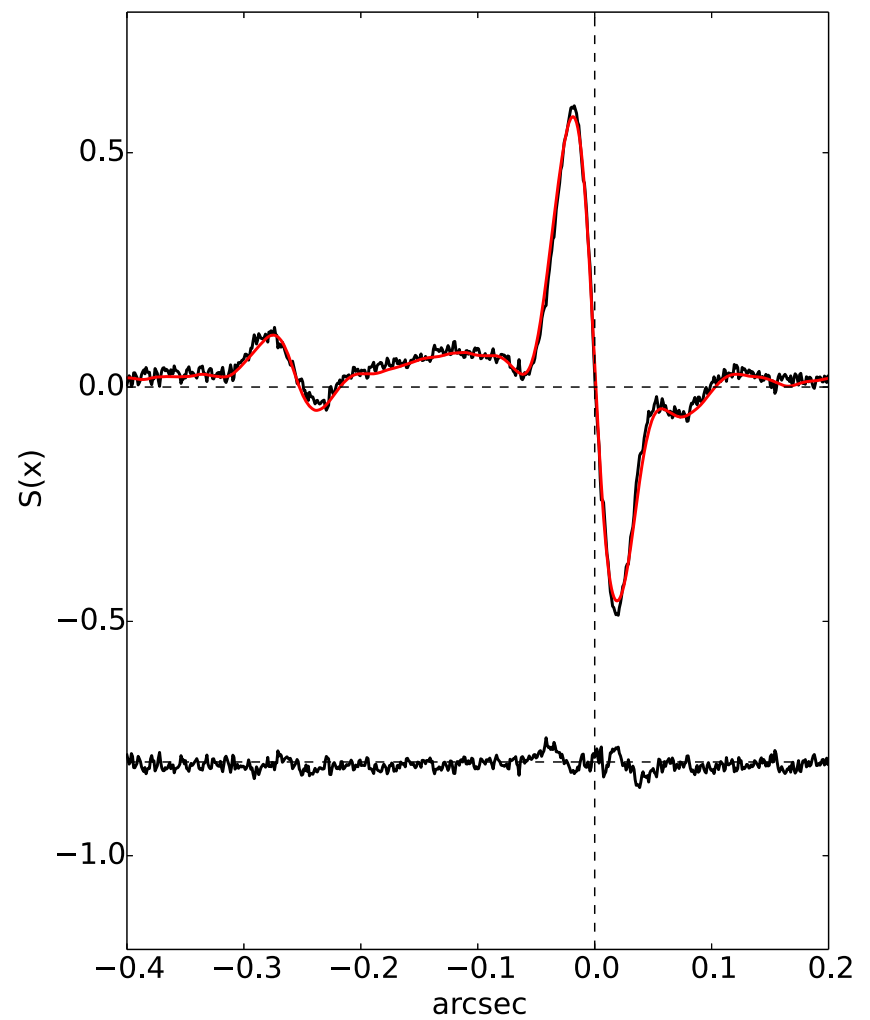

Figure 3. The "S-curve" along the $X$-axis from a Hubble Space Telescope Fine Guidance Sensor observation of LHS 440 AB. The black line on the top represents the data and the red line is the best fit. The residuals to the fit are shown on the bottom of the plot. The secondary is clearly seen at a separation of 256 mas with a $\Delta_{F 583 W}=2.03$. Note that the $X$-axis is not the direction of R.A., because of the HST's roll angle at the observation epoch. The binary is not resolved along the $Y$-axis, so it is not shown here.

both USNO and CTIO parallax programs use " $P$ ' filters, their bandpasses are different. The effective central wavelength and filter bandwidth for USNO and CTIO are $8074 \AA / 1890 \AA$ and $8118 \AA / 1415 \AA$, respectively. These two different bandpasses may cause the slight difference in the photocentric semimajor axis.

Schilbach et al. (2009) reported a parallax of $33.1 \pm 1.32$ mas, and an updated USNO parallax of $30.73 \pm 0.34$ mas, calculated using a Markov chain Monte Carlo simulation, is given in Koren et al. (2016). After removing the perturbation of the photocenter shown in Figure 2(a), we find a parallax of $32.26 \pm 0.48$ mas. The final weighted mean parallax from these three measurements is $31.32 \pm 0.27$ mas.

1718-4326 (LHS $440 A B$ ). Jao et al. (2008) reported this to be a M1.0 subdwarf and Jao et al. (2011) first reported the possible unseen companion based upon a photocentric perturbation. We have extended the coverage from nine years in Jao et al. (2011) to $\sim 15$ years in this work, yielding the perturbation curve shown in Figure 2(b). Because of the very long orbital period for the system, we do not detect a full photocentric orbit yet. The parallax of $39.56 \pm 1.02$ mas presented in Table 1 has had the perturbation removed and supersedes previously reported values in Jao et al. (2005) and Jao et al. (2011).

We used the FGS1r (Fine Guidance Sensors) on the Hubble Space Telescope to resolve this system in Cycle 16 on 2009 April 10th, with scan duration of $1301 \mathrm{~s}$ and scan lengths of $6^{\prime \prime}$. Observations were made through the $F 583 W$ filter, which

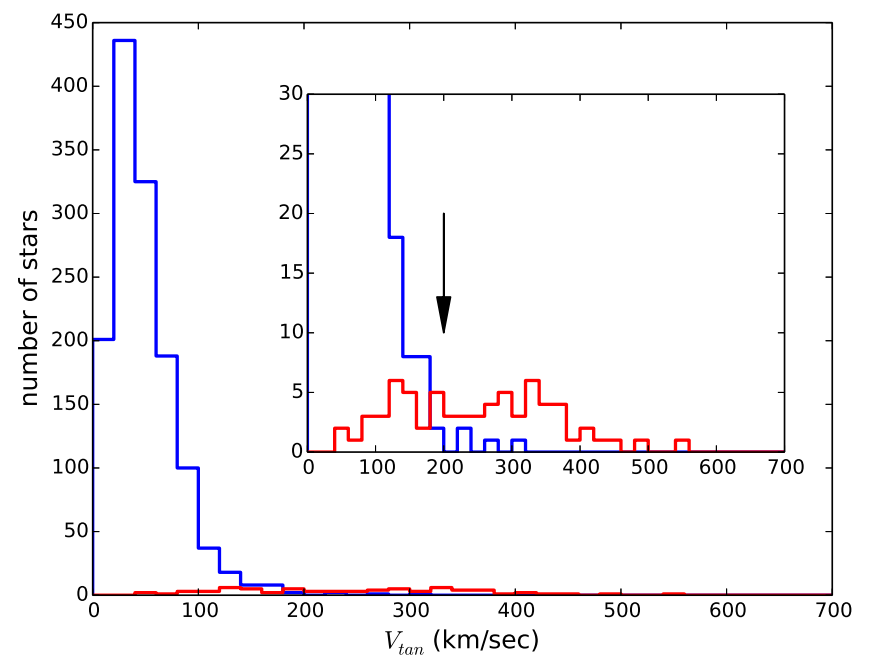

Figure 4. A histogram of tangential velocities for stars in different stellar populations. $\mathrm{K}$ and $\mathrm{M}$ type stars within $25 \mathrm{pc}$ are represented with the blue line, with a peak near $30 \mathrm{~km} \mathrm{~s}^{-1}$. Tangential velocities of 167 subdwarfs from our work and the literature are shown in red. The inset panel shows a zoomed plot. The arrow marks the $200 \mathrm{~km} \mathrm{~s}^{-1}$ limit we use to select $\mathrm{K}$ and $\mathrm{M}$ subdwarfs that are found at larger tangential velocities. The four stars with $V_{\tan }>200 \mathrm{~m} \mathrm{~s}^{-1}$ shown in blue are known subdwarfs within 25 pc.

provides magnitude differences similar to the $V$ band in the Johnson system. The standard strfits routine in the IRAF/ STSDAS package was used to measure the separation, position angle, and magnitude difference between the two components. Additional details of the reduction procedure can be found in the HST/FGS Data Handbook http://www.stsci.edu/hst/fgs/ documents/datahandbook/. The calibrator is LHS 73, which is also a subdwarf and was observed in the same HST observing Cycle. LHS 440 AB was successfully resolved along the $X$-axis, as shown in Figure 3. The pair was not resolved along the $Y$-axis at this epoch, indicating a separation of \pm 10 mas, so we cannot calculate the companion's separation and position angle. The magnitude difference is $2.03 \mathrm{mag}$ in the $F 583 \mathrm{~W}$ filter. Henry et al. (1999) presented a conversion from $\Delta m_{F 583 W}$ to $\Delta V$ using $B-V$ colors, but no $B$ photometry is available for the components, and the relations used in the absence of $B$ photometry apply to dwarfs rather than subdwarfs. Assuming for now that $\Delta V \approx 2$, we find $M_{V}=11.13$ and 13.13 for the components. From the mass-luminosity relation for main sequence red dwarfs of Benedict et al. (2016), this implies masses of 0.33 and $0.19 M_{\odot}$, but again, because these are subdwarfs rather than dwarfs, we emphasize that these should be considered only crude estimates.

1750-5636 (LHS 456). YPC provides a parallax of $39.2 \pm 12.6$ mas and we find $39.87 \pm 1.43$, resulting in a weighted mean value of $39.86 \pm 1.42$ mas.

1809-6154 (SCR1809-6154 AB). The separation of these two stars is $19 ! \prime 2$ at a position angle of $270^{\circ} .6$. Because the primary star is very close to a background star, no variability is reported for the primary and only frames with seeing less than 1 ." 4 were kept for the astrometric reduction. The weighted mean parallax for the two components is $5.50 \pm 1.16$ mas.

$1809+2755(G 182-41 A B)$. This is a double-lined spectroscopic binary with $[\mathrm{Fe} / \mathrm{H}]=-1.0$ (Goldberg et al. 2002), clearly indicating that it is a subdwarf. Our parallax of $9.96 \pm 2.33$ mas places the pair at $\sim 100 \mathrm{pc}$, indicating that the resolution needed to determine masses will prove difficult. 
$2101+0307$ (USN 2101+0307AB). The combined photometry make this system elevated on the $\mathrm{H}-\mathrm{R}$ diagram. We do not have a spectrum for this system. Because of its location on the H-R diagram, we temporarily assign its luminosity class as "V."

2239-3615 (LHS 3841AB). Friedrich et al. (2000) showed this star to have the combined spectra of a helium-rich white dwarf and an $\mathrm{M}$ dwarf using a spectrograph with a coverage of 3800-9200 ̊. Reid \& Gizis (2005) later identified the red dwarf to be an M2.5 subdwarf with a wavelength coverage of $6200-7500 \AA$ and estimated the distance to be 19 pc. However, we determine a parallax of $12.13 \pm 1.47$ mas, placing the system at $\sim 80 \mathrm{pc}$. The erroneous distance estimate was likely due to excess flux from the white dwarf not being considered. Farihi et al. (2010) used the Advanced Camera for Surveys High-Resolution Camera on the Hubble Space Telescope in an attempt to resolve this system using the $F 814 W$ filter, but LHS3841 AB was not resolved. Over 4.2 years of astrometric observations, we do not detect any perturbation of the photocenter position.

2343-2409 (LHS 72 and LHS 73). Both components are subdwarfs (Rodgers \& Eggen 1974; Reylé et al. 2006; Jao et al. 2008), separated by $1 ! 6$ at position angle of $153^{\circ}$. The primary, LHS 72, has a parallax of $37.6 \pm 8.9$ mas in the YPC, but no separate parallax is given for LHS 73. We find parallaxes of $35.20 \pm 1.63$ mas and $32.73 \pm 1.44$ mas for the primary and secondary, respectively, which agree within $2 \sigma$. The weighted mean of all three parallax measurements is $33.87 \pm 1.07$ mas.

\section{Discussion: Observational Differences between Dwarfs and Subdwarfs}

Subdwarfs are low-metallicity stars that have historically been discovered through proper motion surveys, spectroscopic surveys, or color index searches. Historically, the extensive proper motion surveys by Luyten and Giclas have been the primary sources for finding subdwarfs (Bessell 1982; Ryan \& Norris 1991; Gizis 1997). Recent new proper motion surveys with fainter magnitude limits like SuperBLINK (Lépine \& Shara 2005), SuperCOSMOS-RECONS (Subasavage et al. 2005), and SIPS (Deacon et al. 2005) have discovered many new metal-deficient high proper motion subdwarf candidates. To confirm that these candidates are, indeed, cool subdwarfs, spectroscopic observations are typically necessary, such as those by (Scholz et al. 2004b; Lépine et al. 2007; Jao et al. 2008; Zhang et al. 2013). Large sky spectroscopic surveys like SDSS and LAMOST have also provided systematic ways to identify cool subdwarfs (West et al. 2004; Zhong et al. 2015). Finally, because subdwarfs have different colors from dwarfs, infrared colors from the all sky infrared surveys 2MASS and WISE have been used to identify local stellar and sub-stellar subdwarfs (Burgasser et al. 2007; Kirkpatrick et al. 2011).

Here we discuss two other observational methods that allow the identification of cool subdwarf candidates among stars in the solar neighborhood. These methods utilize the astrometry and photometry data presented in this paper to evaluate tangential velocities (using astrometry only), and positions on the H-R diagram (using both astrometry and photometry) to reveal cool subdwarfs. After outlining how subdwarfs can be identified, we apply these two methods on samples from Gaia DR1 and the MEarth Project to identify new nearby subdwarfs.

\subsection{Tangential Velocity}

Kinematic methods that map the motions of stars in our Galaxy have been used to identify subdwarfs because over billions of years, old, low-metallicity subdwarfs are generally disk-heated to higher spatial velocities. The challenge in using $U V W$ kinematics to separate subdwarfs from dwarfs is that both trigonometric parallaxes and radial velocities are required for each candidate star. Nonetheless, several efforts have revealed trends. Ryan \& Norris (1991) found that, while $\sigma_{U}, \sigma_{V}$ and $\langle V\rangle$ are independent of metallicity, the vertical velocity dispersion, $\sigma_{W}$, increases with decreasing metallicity. They use $\sigma_{W}$ values to separate over 770 FGKM stars from the NLTT catalog, among which they flagged $115 \mathrm{~K}$ and $4 \mathrm{M}$ subdwarfs. A study by Arifyanto et al. (2005), based on 742 nearby metal-poor stars from Carney et al. (1994), reported that halo stars with $[\mathrm{Fe} / \mathrm{H}]<-1.6$ have a low mean rotational velocity around the Galaxy and a radially elongated velocity ellipsoid, while stars with $-1.6<[\mathrm{Fe} / \mathrm{H}]<-1$ have disk-like kinematics. Based on a limited sample of 69 subdwarfs with both parallaxes and radial velocities, Gizis (1997) found different mean Galactic rotation velocities between different sub-types of subdwarfs and noted that, overall, subdwarfs move faster than regular $\mathrm{M}$ dwarfs. A recent result by Savcheva et al. (2014), using a much larger sample (3517) drawn from SDSS data, showed the same trend as Gizis (1997).

Without radial velocities, many authors have turned to using a tangential velocity cutoff to select subdwarfs in lieu of complete $U V W$ motions. As a benchmark, Hawley et al. (1996) reported that a northern sample of $514 \mathrm{M}$ dwarfs has an average tangential velocity of $43.8 \mathrm{~km} \mathrm{~s}^{-1}$. In order to get an uncontaminated sample of halo stars from the Giclas high proper motion survey, Schmidt (1975) imposed a hard limit on tangential velocity of $250 \mathrm{~km} \mathrm{~s}^{-1}$ to determine the luminosity function of halo stars. Later, Gizis \& Reid (1999) used various tangential velocity cutoffs to select halo stars from the reduced proper motion diagram in order to revise the luminosity function of the halo population. Stars having $V_{\tan }>75 \mathrm{~km} \mathrm{~s}^{-1}$ were flagged as $\mathrm{M}$ extreme subdwarf candidates, while stars with $V_{\tan }>125 \mathrm{~km} \mathrm{~s}^{-1}$ were flagged as $\mathrm{M}$ regular subdwarf candidates ${ }^{10}$ Digby et al. (2003) used a tangential velocity of $200 \mathrm{~km} \mathrm{~s}^{-1}$ to select their subdwarf candidates based on the reduced proper motion diagram.

Now that we have parallaxes for a relatively large sample of $\mathrm{K}$ and $\mathrm{M}$ subdwarfs, we use the sample to determine an appropriate $V_{\tan }$ cutoff that can be used to identify subdwarfs. Figure 4 illustrates $V_{\tan }$ distributions of main sequence dwarfs and subdwarfs. The 1324 nearby $\mathrm{K}$ and $\mathrm{M}$ dwarfs with parallaxes placing them within $25 \mathrm{pc}$ were selected using spectral types and a color cutoff of $V-K_{s}>1.9$, and are represented by the blue curve. The red line indicates the $167 \mathrm{~K}$ and $\mathrm{M}$ subdwarfs observed during our CTIOPI program with new or revised parallaxes from RECONS, supplemented with confirmed subdwarfs collected from the literature (Ryan \& Norris 1991; Carney et al. 1994; Hawley et al. 1996; Gizis 1997; Cayrel de Strobel et al. 2001; Burgasser et al. 2003; Lépine et al. 2003a; Woolf \& Wallerstein 2005; Jao et al. 2009;

\footnotetext{
${ }^{10}$ The reduced proper motion, " $H$," is defined as $H=m+5 \log \mu+5=$ $M+5 \log \frac{V_{\tan }}{4.74}$ where $m$ is the apparent magnitude and $\mathrm{M}$ is the absolute magnitude. For a given color, the $\mathrm{M}$ extreme subdwarfs are fainter than regular subdwarfs. Therefore, M extreme subdwarfs do not need to have their $V_{\tan }$ values as high to be placed a few magnitudes below dwarfs on the reduced proper motion diagram.
} 


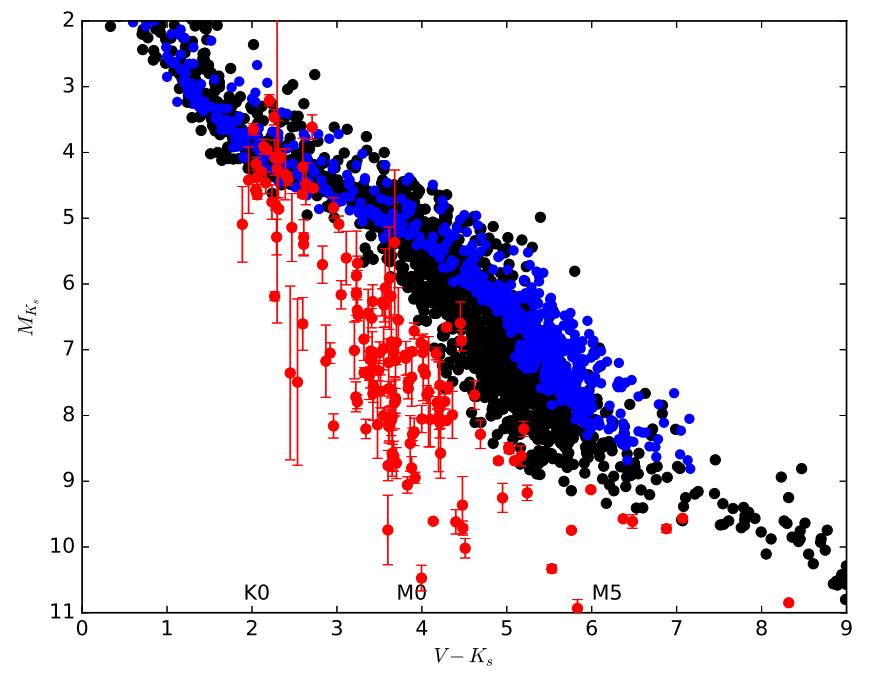

Figure 5. H-R diagram of Pleiades members, nearby stars within $25 \mathrm{pc}$, and subdwarfs. Pleiades (blue points) are from Rebull et al. (2016), with a uniform parallax of $7.45 \pm 0.3$ mas from Gaia DR1 (Gaia Collaboration et al. 2016) assigned to all stars. The $V-K_{s}$ values for the Pleiades members are the dereddened colors from Rebull et al. (2016). Nearby stars (black points) are the same as the gray points in Figure 1 (nearby white dwarfs are beyond the boundaries of this plot). Subdwarfs are shown in red. We omit error bars for the dwarfs and the Pleiades, but their mean absolute magnitude errors are \pm 0.05 and \pm 0.06 , respectively. Errors in the horizontal direction are smaller than the points for all three samples.

Wright et al. 2014). This plot shows that most $\mathrm{K}$ and $\mathrm{M}$ dwarfs in the solar neighborhood have $V_{\tan }<100 \mathrm{~km} \mathrm{~s}^{-1}$, with a peak in the distribution at $30 \mathrm{~km} \mathrm{~s}^{-1}$, consistent with the average tangential velocity discussed in Hawley et al. (1996) near $45 \mathrm{~km} \mathrm{~s}^{-1}$. Adopting a cutoff of $V_{\tan }=200 \mathrm{~km} \mathrm{~s}^{-1}$ reveals only four stars at higher $V_{\tan }$ values, each of which is, in fact, a known nearby subdwarf. Thus, there are no known main sequence $\mathrm{K}$ and $\mathrm{M}$ dwarfs within $25 \mathrm{pc}$ with $V_{\tan }>200 \mathrm{~km} \mathrm{~s}^{-1}$. Unfortunately, this cutoff permits us to identify only $57 \%(95 / 167)$ of (fast-moving) subdwarfs and excludes the remaining $43 \%$ with slower $V_{\tan }$ values. Nonetheless, although solar neighborhood stars with tangential velocities less than $200 \mathrm{~km} \mathrm{~s}^{-1}$ comprise a mixed population of young, middle-aged, and old stars, virtually all nearby stars with tangential velocities greater than $200 \mathrm{~km} \mathrm{~s}^{-1}$ are confirmed subdwarfs. This cutoff may be used with confidence to select samples of cool subdwarfs.

\subsection{Hertzsprung-Russell Diagram}

In Figure 1, we use $M_{V}$ and $\left(V-K_{S}\right)$ to illustrate the locations of the sample of objects targeted here. We show an additional $\mathrm{H}-\mathrm{R}$ diagram in Figure 5 using $V-K_{s}$ versus $M_{K}$, rather than $M_{V}$. Stars within $25 \mathrm{pc}$ on the main sequence are shown in black, overlaid with members of the Pleiades (Rebull et al. 2016) in blue and the 167 subdwarfs discussed in the previous section in red. These three sets of stars of various ages and metallicities are merged blueward of $V-K_{s} \approx 2$ and brighter than $M_{K} \approx 4$. In contrast, the three samples form clear "bands" in this diagram in the redder, fainter portion of the diagram. Based on Figure 5, we conclude that: (1) the general trend shows that the populations' ages, from $\sim 100 \mathrm{Myr}$ for the Pleiades, to mixed ages of a few Gyr for disk stars, to 6-9 Gyr for subdwarfs ${ }^{11}$ ), as well as differing

\footnotetext{
11 Monteiro et al. (2006) measured the ages of two cool subdwarfs based on their white dwarf companions. We adopt this age range as representative of the subdwarfs shown here.
}

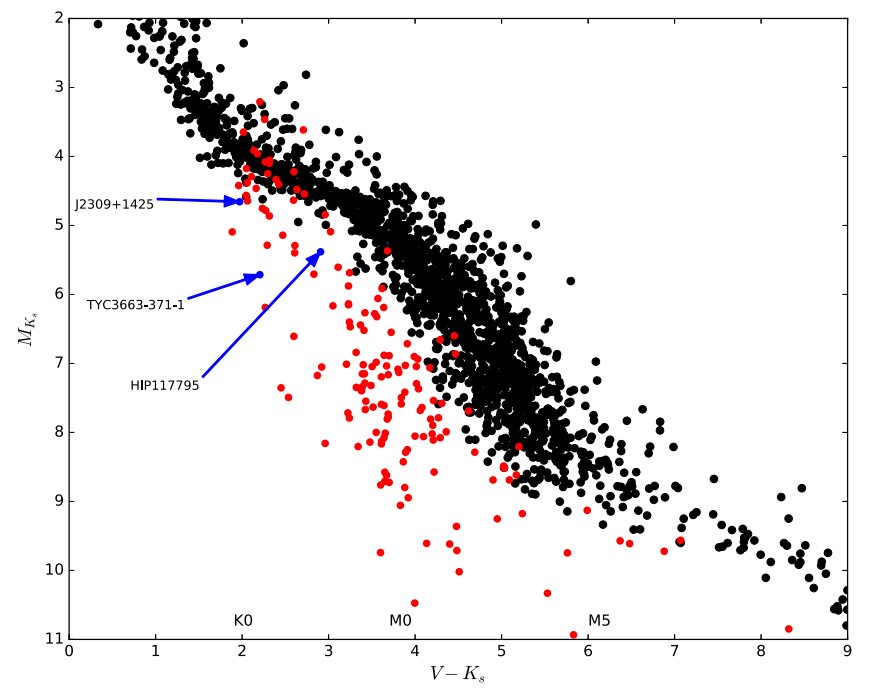

Figure 6. Black points are stars within $25 \mathrm{pc}$ and red points are confirmed subdwarfs. The three labeled blue points are candidate subdwarfs within $60 \mathrm{pc}$ below the main sequence from Gaia DR1 and are discussed in Section 6. The Johnson $V$ magnitudes for these three stars are converted from the Tycho $2 B$ and $V$ magnitudes. After further analysis, only TYC 3663-371-1 remains a subdwarf candidate.

metallicities, causes the shift in the stellar distribution on the H-R diagram. (2) Early K-type young stars, dwarfs, and subdwarfs are indistinguishable in the H-R diagram of Figure 5. (3) There is no prominent void in the distribution of subdwarfs from (at least) $V-K_{S}=2-7$ in the observational H-R diagram. Gizis (1997) identified many cool subdwarfs spectroscopically and established an important foundation in subdwarf studies. He found a void on his H-R diagram that lacked "sdM" subdwarfs with $2.2<$ $V-I<2.8$, corresponding to $3.9<V-K_{s}<5.2$. Although the number of stars in this region is still smaller than at bluer colors, the void has begun to fill in because new subdwarf identification efforts since have increased the number of subdwarfs in this color range. Given that the metallicity distribution is smooth instead of clumped (Gizis 1997), we expect more subdwarfs in this color range should be unveiled in the future.

\section{Subdwarfs Identified using $V_{\text {tan }}$ and the H-R diagram}

We applied these two methods for identifying new subdwarfs to the latest large sets of parallaxes recently released in Gaia DR1 (Gaia Collaboration et al. 2016, GAIADR1), which contains stars in common between the Tycho-2 Catalog and Gaia mission, and the MEarth Project, which focuses on nearby M dwarfs (Dittmann et al. 2014).

\subsection{The Gaia DR1 Catalog}

We extracted 9494 targets within 60 pc from Gaia DR1. Not all stars have Johnson $V$ magnitudes, so a conversion $(V=$ $\left.V_{T}-0.09 *\left(B_{T}-V_{T}\right)\right)$ from the Tycho 2 catalog was used to convert Tycho $2 \mathrm{~V}$ magnitudes to the standard Johnson $V$ filter. None of these 9494 targets has a tangential velocity greater than $200 \mathrm{~km} \mathrm{~s}^{-1}$. We identified only three stars clearly below the main sequence on the HR diagram. These three candidates are plotted in Figure 6 and discussed below. We find that two stars are subdwarf candidates, but the third is not a subdwarf. The paucity of intrinsically faint new subdwarf candidates is 
Table 3

Two Wide Binaries Selected from Gaia DR1

\begin{tabular}{|c|c|c|c|c|c|c|}
\hline Name & $\begin{array}{c}\pi \\
\operatorname{mas}\end{array}$ & Ref & $\begin{array}{c}\text { Tycho } 2 \mu_{\mathrm{R} . \mathrm{A}} . \\
\text { mas }\end{array}$ & $\begin{array}{c}\text { Tycho } 2 \mu_{\text {Decl. }} \\
\text { mas }\end{array}$ & $\begin{array}{c}\text { Gaia } \mathrm{DR} 1 \mu_{\mathrm{R} . \mathrm{A} .} \\
\text { mas }\end{array}$ & $\begin{array}{c}\text { Gaia } \operatorname{DR} 1 \mu_{\text {Decl. }} \\
\text { mas }\end{array}$ \\
\hline HD $4868^{a}$ & $16.28 \pm 0.79$ & Hipparcos & $62.2 \pm 1.5$ & $-80.9 \pm 1.5$ & $\ldots$ & $\ldots$ \\
\hline TYC $3663-371-1^{a}$ & $21.84 \pm 0.81$ & Gaia DR1 & $69.2 \pm 5.8$ & $-88.7 \pm 5.8$ & $46.43 \pm 1.84$ & $-66.98 \pm 1.57$ \\
\hline HIP $114378^{a}$ & $39.87 \pm 0.41$ & Gaia DR1 & $-121.7 \pm 4$ & $-84.8 \pm 5$ & $-121.55 \pm 0.04$ & $-85.36 \pm 0.03$ \\
\hline TYC $1167-683-1^{a}$ & $36.55 \pm 0.75$ & Gaia DR1 & $-125.8 \pm 13$ & $-96.3 \pm 14$ & $-107.25 \pm 0.80$ & $-91.25 \pm 0.86$ \\
\hline
\end{tabular}

Note.

${ }^{\mathrm{a}}$ We find that neither of these two systems form a binary.

not surprising, given that only stars bright enough to be observed by Tycho have parallaxes in Gaia DR1.

$(0051+5629)$ TYC 3663-371-1. This star is almost two magnitudes fainter than stars in the center of the main-sequence band in Figure 6, providing strong evidence that it is a $\mathrm{K}$ subdwarf. No metallicity measurement is found in the literature.

The star is listed as a companion to a $G$ star (HD 4868) in SIMBAD, and The Washington Double Star Catalog lists the two stars as a visual binary (WDS $00515+5630 \mathrm{AB}$ ) with a separation of 40".6 (Høg et al. 2000). Gaia DR1 reports a parallax of $21.84 \pm 0.81$ for TYC 3663-371-1, but no parallax for HD 4868. HD 4868 does have a parallax of $16.28 \pm 0.79$ in the Hipparcos catalog (van Leeuwen 2007), which is $\sim 5 \sigma$ offset from TYC 3663-371-1's value. Both stars have similar proper motions in Tycho 2, but the proper motion for TYC 3663-371-1 in Gaia DR1 (see Table 3) is very different. Because of the parallax and proper motion differences, we do not believe these two stars form a physically bound system.

$(2309+1425)$ LSPM J2309+1425 = TYC 1167-683-1. There is an X-ray source in the direction of this star, which is found in a region of high Galactic latitude molecular clouds (Li et al. 2000). The ROSAT catalog shows an X-ray source with $f_{x} / f_{\text {opt }}=-1.93$ for this star and $\mathrm{Li}$ et al. (2000) reported detection of $\mathrm{H} \alpha$ emission. Cutispoto et al. (2002) even set an upper limit on the lithium equivalent width of 0.8 milli-Angstrom. The lithium line, $\mathrm{X}$-ray, and $\mathrm{H} \alpha$ emission typically indicate youth, so this star is not a subdwarf. In addition, as shown in Figure 6, stars of different ages merge at this color on the $\mathrm{H}-\mathrm{R}$ diagram, and this star is barely offset from main sequence stars.

The star is listed as a companion to HIP 114378 in SIMBAD, and The Washington Double Star Catalog lists the two stars as a visual binary (WDS $23100+1426 \mathrm{AB}$ ) with a separation of 31 !"7 (Høg et al. 2000). Both stars have parallaxes and proper motions in Gaia DR1, given in Table 3. As with TYC 3663-371-1, the distances and proper motions given in Table 3 do not support that the two components are physically bound. Their parallaxes differ by $\sim 4 \sigma$ and the proper motions in Gaia DR1 do not match. Hence, these two stars are likely not associated.

$(2353+5956)$ HIP 117795. This star has no metallicity measurement in the literature. Sperauskas et al. (2016) reported a radial velocity of $-285.9 \pm 0.2 \mathrm{~km} \mathrm{~s}^{-1}$. Although this star's tangential velocity $\left(11.2 \mathrm{~km} \mathrm{~s}^{-1}\right)$ is less than $200 \mathrm{~km} \mathrm{~s}^{-1}$, the Gaia parallax (37.49 \pm 0.23 mas) and proper motion, combined with the radial velocity measurement, yield $U, V$, $W=(-114,-262,+12)$. Thus, kinematically, HIP 117795 is very different from nearby disk M dwarfs (Hawley et al. 1996) and it is about one magnitude below the center of the main sequence in Figure 6. As we discussed earlier, Arifyanto et al. (2005) showed halo stars with $[\mathrm{Fe} / \mathrm{H}]<-1.6$ have low mean galactic rotational velocity, but their velocities range from +200 to $-200 \mathrm{~km} \mathrm{~s}^{-1}$. As for stars with $[\mathrm{Fe} / \mathrm{H}]>-1.0$ in Arifyanto et al. (2005), their galactic rotational velocities are clustered around $+200 \mathrm{~km} \mathrm{~s}^{-1}$. The direction and velocity of HIP 117795 's $V$ indicate that it has a retrograde motion and is much faster than the limit shown in Arifyanto et al. (2005) for halo stars. By combining its kinematic and location on the $\mathrm{H}-\mathrm{R}$ diagram, we deduce that it is likely a nearby $\mathrm{K}$ subdwarf.

\subsection{The MEarth Project}

The MEarth Project released 1507 parallaxes of nearby M dwarfs (Dittmann et al. 2014), but after taking companions into account, there are a total of 1511 entries. Although this list of stars lacks spectral classifications, most of them should be $M$ dwarfs, as MEarth is targeting M dwarfs. After calculating each star's tangential velocity, we find that only LSPM J2107+5943 (LHS 64) has $V_{\tan }>200 \mathrm{~km} \mathrm{~s}^{-1}$, and it is a known nearby cool subdwarf (Gizis 1997).

To reveal additional subdwarfs, we also wish to check the locations of these stars on the H-R diagram. (Dittmann et al. 2014) provide only 2MASS $J$ and $K_{s}$ magnitudes, and unlike the $V-K_{s}$ color shown in Figure $5, J-K_{s}$ does not clearly differentiate young $M$ dwarfs, disk $M$ dwarfs, and $M$ subdwarfs on the H-R diagram. In addition, not all 1511 stars have Johnson $V$ magnitudes, so it is not possible to overplot the entire sample on the same scale as shown in Figure 5 for comparison.

In order to create a set of uniform photometry, we crossmatched the stars with entries in the recent PanSTARRS data release (Flewelling et al. 2016) and extracted $g$ and $r$ magnitudes. In the PanSTARRS data release, high proper motion stars usually, but not always, have multiple entries and coordinates because of the different epochs of PanSTARRS images, resulting in several lines of matches for a given coordinate and search radius. Presumably, all of these entries are the same star moving across the sky. On the other hand, if a star has only one correct entry in PanSTARRS, we may also get multiple lines because of several sources within the search radius. In order to separate correct matches from false ones, we apply multiple steps to extract PanSTARRS photometry. First, we submitted MEarth stars' equinox and epoch J2000 coordinates to the PanSTARRS site (http://archive.stsci.edu/panstarrs/search.php) and set the search radius to 0.5. We calculated the mean epoch from all entries found in this search. We then slid the MEarth stars' coordinates from J2000 to that mean epoch using the MEarth proper motions. The second search was done by querying these new coordinates with a reduced search radius of 0.1 , and "PSFMag" values were extracted. Most stars have only one match in the PanSTARRS catalog at this mean epoch with the smaller search radius, but for those stars with multiple matches, a 

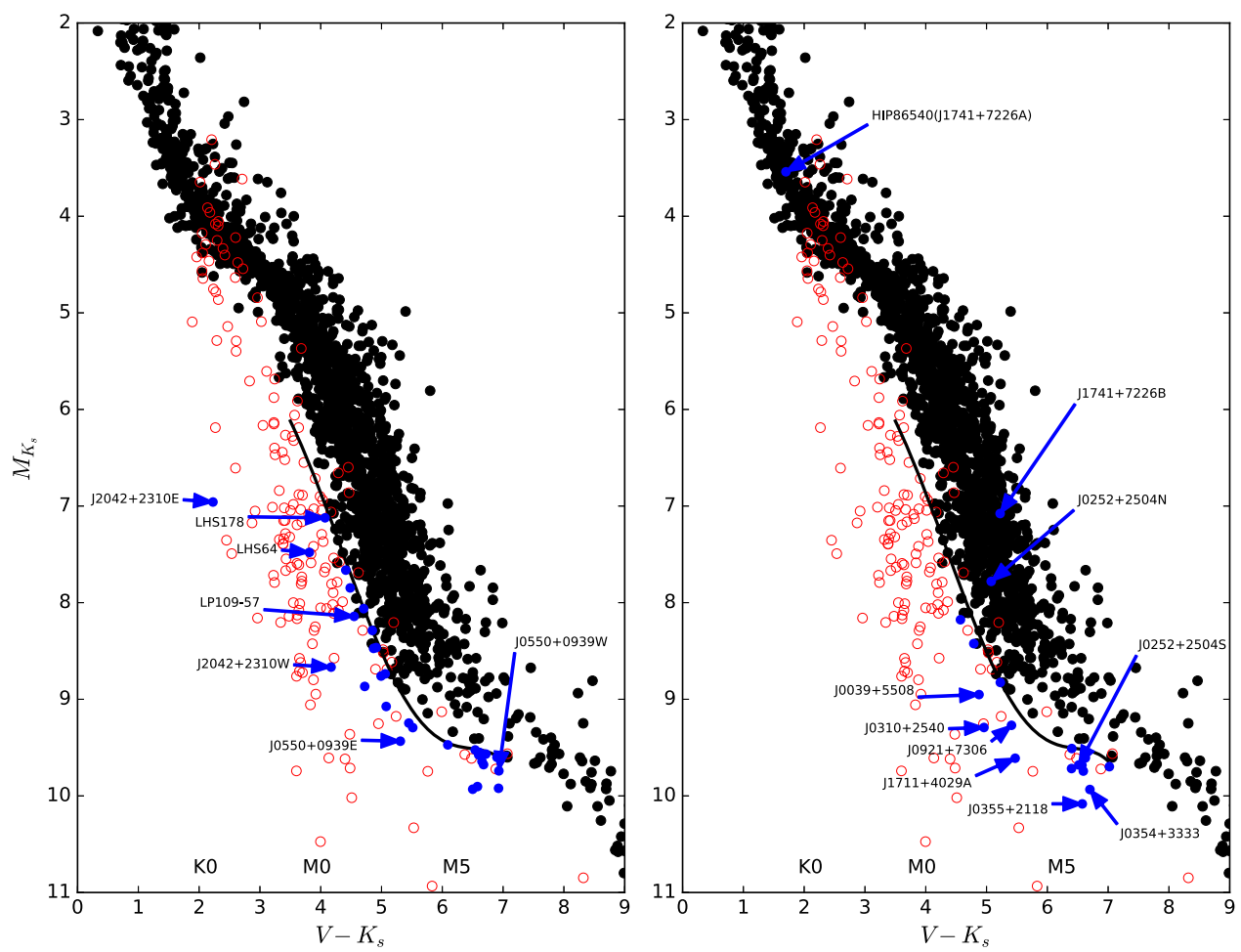

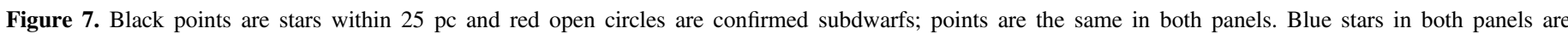

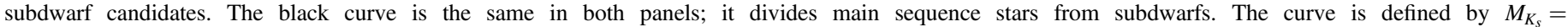

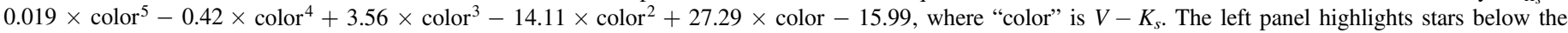

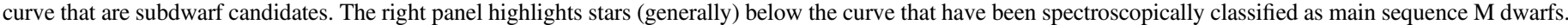
Labeled stars are discussed in Section 6.

mean photometric magnitude at a given filter was calculated, with its standard deviation required to be less than $0.5 \mathrm{mag}$ to eliminate mixing background sources-individual photometric measurements for a given filter typically have errors less than 0.04 mag. In total, 1349 stars had both $g$ and $r$ magnitudes that were then converted to Johnson $V$ magnitudes using the equation $V=g-0.5784 *(g-r)-0.0038$, which is available at SDSS's website (http://www.sdss3.org/dr8/algorithms/sdss UBVRITransform.php\#West2005). We note that PanSTARRS filter bandpasses differ slightly from those of the SDSS filters.

Rather than relying on our converted $V$ magnitudes, we choose to identify possible subdwarfs empirically by using cutoffs in $M_{K s}$ instead of $M_{V}$ because the 2MASS $K_{s}$ photometry is uniformly consistent. The fifth-order polynomial line shown in Figure 7 was established based on where known subdwarfs (represented with red points) are found relative to main sequence stars (black points). The 51 MEarth stars located on or below this dividing line are considered to be subdwarf candidates and are listed in Table 4. Three highlighted stars above the lines in the two panels of Figure 7 are discussed below.

Among these 51 stars are 30 subdwarf candidates shown with blue points in the left panel of Figure 7. Blue points in the right panel represent 20 stars that have been spectroscopically identified as regular dwarfs in the literature and one (LSPM $\mathrm{J} 1012+2113$ ) that is a close double with a suspect position in Figure $7 .{ }^{12}$

\footnotetext{
${ }^{12}$ LSPM J1012+2113EW was initially identified as a subdwarf candidate and single star, but was flagged by MEarth as having a nearby bright contaminating star (Newton et al. 2016). PanSTARRS resolves it as a binary star separated by $\sim 2$. 0 at $\sim 93^{\circ} .3$ with $\Delta g=0.01$. Without individual $K_{s}$ magnitudes or spectral types, we cannot estimate its luminosity class at this time.
}

\subsubsection{New Subdwarf Candidates}

Among the 30 subdwarf candidates selected using this method, LHS 64, LHS 178, and LP 109-57 are previously identified M subdwarfs and labeled in the left panel of Figure 7. Thus, we present here 27 new subdwarf candidates, listed in Table 4 . We identify two wide subdwarf binary systems, LSPM J0550+0939EW and LSPM J2042+2310EW, each with both components falling below the dividing line and labeled in Figure 7. In particular, LSPM J2042+2310E is $\sim 3$ full magnitudes below the main sequence.

\subsubsection{Spectroscopically Confirmed M Dwarfs}

Twenty stars in 17 systems shown in the right panel of Figure 7 were previously identified as $M$ dwarfs in the literature. Several of these stars are worth discussing to outline why this group of stars should not be considered subdwarfs.

LSPM J1741+7226B is an M4 dwarf (Alonso-Floriano et al. 2015) located in the center of main-sequence in Figure 7. It is a wide common proper motion companion to a bright star, HIP 86540/G 258-16A (Lépine \& Bongiorno 2007). The MEarth parallax is $77.6 \pm 5.0$ mas for LSPM J1741+7226B, so initially this star was identified as a subdwarf candidate using the H-R diagram. However, Gaia DR1 reports a parallax of $33.17 \pm 0.21$ mas for the primary, so the weighted mean parallax moves LSPM J1741+7226B upward to the current location shown in Figure 7. This revised location on the H-R diagram matches its luminosity, as classified by AlonsoFloriano et al. (2015).

Six stars-LSPM J0039+5508 (LHS 6009), LSPM J0310 +2540, LSPM J0354+3333, LSPM J0355+2118, LSPM 
Table 4

Subdwarf Candidates Selected from MEarth

\begin{tabular}{|c|c|c|c|c|c|c|c|c|c|c|}
\hline R.A. & Decl. & Name1 & Name2 & $g$ & $r$ & $V$ & $V-K_{s}$ & $M_{K s}$ & SpT & References \\
\hline \multicolumn{11}{|c|}{ Previously Identified as Subdwarfs } \\
\hline 034230.06 & +123116.20 & LSPM J0342+1231 & LHS 178 & 13.55 & 12.52 & 12.95 & 4.07 & 7.12 & $\operatorname{sdM} 1.5$ & \\
\hline 225815.55 & +614426.30 & LSPM J2258+6144 & LP $109-57$ & 14.78 & 13.46 & 14.01 & 4.55 & 8.14 & sdM3 & \\
\hline \multicolumn{11}{|c|}{ New Subdwarf Candidates } \\
\hline 014154.90 & +384325.00 & LSPM J0141+3843 & & 15.60 & 14.32 & 14.85 & 4.91 & 8.47 & & \\
\hline 014353.38 & +001431.10 & LSPM J0143+0014 & & 17.93 & 16.60 & 17.16 & 6.90 & 9.60 & & \\
\hline 030535.80 & +193406.80 & LSPM J0305+1934 & & 15.57 & 14.37 & 14.87 & 4.99 & 8.76 & & \\
\hline 031412.77 & +284030.10 & LSPM J0314+2840 & LHS 1516 & 17.47 & 16.12 & 16.69 & 6.60 & 9.58 & & \\
\hline 033622.62 & +135038.90 & LSPM J0336+1350 & LHS 1568 & 15.52 & 14.37 & 14.85 & 4.85 & 8.29 & & \\
\hline 041925.55 & +381501.50 & LSPM J0419+3815 & & 14.80 & 13.54 & 14.07 & 4.71 & 8.06 & & \\
\hline 055011.21 & +094003.70 & LSPM J0550+0940W & & 18.38 & 16.99 & 17.57 & 6.93 & 9.74 & & \\
\hline 063755.42 & +085855.10 & LSPM J0637+0858 & & 16.45 & 15.18 & 15.71 & 5.45 & 9.25 & & \\
\hline 073129.26 & +024908.90 & LSPM J0731+0249 & & 17.63 & 16.30 & 16.85 & 6.61 & 9.55 & & \\
\hline 080121.10 & +562400.40 & LSPM J0801+5624 & & 18.28 & 16.91 & 17.48 & 6.65 & 9.64 & & \\
\hline 091707.19 & +200751.30 & LSPM J0917+2007 & G $41-30$ & 15.32 & 14.19 & 14.66 & 5.06 & 8.74 & & \\
\hline 091920.07 & +215428.40 & LSPM J0919+2154 & LP $369-27$ & 18.86 & 17.49 & 18.07 & 6.72 & 9.57 & & \\
\hline 115958.94 & +210459.90 & LSPM J1159+2105 & LP $375-69$ & 17.46 & 16.20 & 16.73 & 6.09 & 9.47 & & \\
\hline 165105.15 & +780923.10 & LSPM J1651+7809 & LHS 3247 & 17.77 & 16.45 & 17.00 & 6.54 & 9.52 & & \\
\hline 174726.14 & +284038.10 & LSPM J1747+2840 & LHS 6325 & 15.57 & 14.28 & 14.82 & 5.08 & 9.07 & & \\
\hline 175700.01 & +785950.90 & LSPM J1756+7859 & & 18.71 & 17.40 & 17.95 & 6.58 & 9.90 & & \\
\hline 191245.12 & +394320.20 & LSPM J1912+3943 & & 15.29 & 14.08 & 14.58 & 4.72 & 8.87 & & \\
\hline 203209.80 & +601816.80 & LSPM J2032+6018 & & 15.04 & 13.79 & 14.31 & 4.41 & 7.66 & & \\
\hline 204229.08 & +231013.50 & LSPM J2042+2310E & & 11.20 & 10.60 & 10.85 & 2.23 & 6.96 & & \\
\hline 025233.27 & +250446.70 & LSPM J0252+2504N & G $36-39 A$ & 15.48 & 14.30 & 14.80 & 5.08 & 7.78 & M4.5 & \\
\hline 025234.18 & +250433.10 & LSPM J0252+2504S & G $36-39 B$ & 18.90 & 17.59 & 18.14 & 6.52 & 9.68 & $\ldots$ & \\
\hline $\begin{array}{lll}03 & 1038.98\end{array}$ & +254051.00 & LSPM J0310+2540 & LP $355-32$ & 14.85 & 13.62 & 14.14 & 4.96 & 9.29 & M3 & \\
\hline 035401.36 & +333321.40 & LSPM J0354+3333 & & 18.39 & 17.07 & 17.62 & 6.70 & 9.93 & M6 & \\
\hline 035537.16 & +211847.60 & LSPM J0355+2118 & LP $357-206$ & 18.44 & 17.13 & 17.67 & 6.57 & 10.08 & M5 & \\
\hline 072918.83 & +755358.80 & LSPM J0729+7554 & LP 1744 & 18.45 & 17.20 & 17.72 & 6.59 & 9.74 & M5.5 & \\
\hline 092022.75 & +264336.20 & LSPM J0920+2643 & LHS 266 & 16.30 & 14.95 & 15.52 & 5.23 & 8.83 & M4.5 & \\
\hline 092116.79 & +730634.20 & LSPM J0921+7306 & LHS 2126 & 15.72 & 14.36 & 14.93 & 5.41 & 9.27 & M4.5 & \\
\hline 135700.62 & +083009.80 & LSPM J1357+0830 & LHS 2828 & 18.31 & 16.99 & 17.54 & 6.40 & 9.51 & M5.5 & \\
\hline 163701.25 & +353538.40 & LSPM J1637+3535 & LHS 3227 & 17.41 & 16.08 & 16.64 & 6.40 & 9.72 & M6 & \\
\hline 171146.38 & +402902.60 & LSPM J1711+4029A & G $203-50 A$ & 16.50 & 15.19 & 15.74 & 5.47 & 9.61 & M4.5 & \\
\hline 171146.38 & +402902.60 & LSPM J1711+4029B & G 203-50B & $\ldots$ & $\ldots$ & $\ldots$ & $\ldots$ & $\ldots$ & $\mathrm{L} 4$ & \\
\hline 174106.69 & +722513.24 & LSPM J1741+7225A & G $258-16$ & $\ldots$ & $\ldots$ & 7.61 & 1.70 & 3.54 & K0 & 10 \\
\hline 174115.76 & +722634.80 & LSPM J1741+7226B & G $258-17$ & 15.39 & 14.14 & 14.66 & 5.22 & 7.08 & M4.0 & 11 \\
\hline 184147.82 & +242150.80 & LSPM J1841+2421 & & 18.16 & 16.83 & 17.39 & 6.63 & 9.61 & M6.0 & \\
\hline 225614.09 & +681532.70 & LSPM J2256+6815 & LHS 3877 & 15.33 & 14.11 & 14.62 & 4.57 & 8.18 & M3.5 & \\
\hline
\end{tabular}

Binary, Presumed Main Sequence Dwarfs

\begin{tabular}{rllllllllll}
\hline 101258.30 & +21 & 13 & 22.20 & LSPM J1012+2113EW & 99.13 & 15.86 & 50.97 & 39.82 & 8.91 & $\ldots$
\end{tabular}

References. (1) Gizis \& Reid (1997), (2) Hawley et al. (1996), (3) Cruz \& Reid (2002), (4) Reid \& Gizis (2005), (5) Reid et al. (2004), (6) Scholz et al. (2005), (7) Lépine et al. (2003b), (8) Cruz \& Reid (2002), (9) Radigan et al. (2008), (10) White et al. (2007), (11) Alonso-Floriano et al. (2015). 
J0921+7306 (LHS2126), and LSPM J1711+4029A (G203$50 \mathrm{~A})^{13}$-highlighted in the right panel of Figure 7 , are well below the main sequence, implying that they are subdwarfs, but their locations contradict their reported spectral types. Their 2MASS $K_{s}$ magnitudes should be reliable, so either their converted $V$ magnitudes or MEarth parallaxes are in error. For example, LSPM J0039+5508 has Johnson $V=14.17$ from Weis (1988), resulting in $V_{J}-K_{s}=4.93$ compared to $V_{\text {converted }}-K_{s}=4.88$. The $0.05 \mathrm{mag}$ difference is not sufficient to relocate this star horizontally onto the main sequence, so if the star is indeed on the main sequence, the parallax is incorrect. Thus, we suspect that the parallaxes for these six stars also need to be revised, as is the case for LSPM $\mathrm{J} 1741+7226$.

LSPM J0252+2504N (G36-39) and J0252+2504S form a wide common proper motion pair. The primary star is clearly on the main sequence and has a spectral type of M4.5 (Reid et al. 2004). Even though the secondary is below our empirical dividing line, it should be considered a dwarf. Thus, several of the stars very near the cutoff line are likely just misplaced in the diagram because of slightly incorrect $V$ estimates or parallaxes.

\section{The Future of Finding Nearby Subdwarfs}

In the past 20 years, thousands of subdwarfs have been identified through all-sky spectroscopic surveys or targeted individual spectroscopic observations. However, without trigonometric parallaxes, we cannot pinpoint their locations on the H-R diagram and link them to their originally defined character: "dwarfs below the main sequence" (Kuiper 1939). Since Bessel measured the first parallax in 1838 for 61 Cygni, trigonometric parallaxes continue to be one of the most essential measurements in stellar astrophysics. In this paper, we contribute parallaxes for 51 systems, including 37 systems for which these are the first parallaxes, of which 15 have proper motions of at least $1^{\prime \prime} \mathrm{yr}^{-1}$. We find that most of the stars targeted here turn out to be cool subdwarfs.

We describe two reliable methods for revealing subdwarfs in the solar neighborhood. By using astrometry alone, we find that, if a nearby $\mathrm{K}$ or $\mathrm{M}$ dwarf has a tangential velocity greater than $200 \mathrm{~km} \mathrm{~s}^{-1}$, it is almost certainly a subdwarf. Using accurate trigonometric parallaxes and $V$ and $K_{s}$ photometry, such as that presented here, we show that, by carefully placing stars on the H-R diagram, we can also identify cool subdwarfs. In the next a few years, the Gaia and LSST efforts will measure countless high-precision parallaxes, proper motions, and photometric values for stars throughout the sky. We plan to apply these two methods to identify a large number of nearby low-metallicity subdwarfs, so that we can unveil more of the missing Galactic relics in the solar neighborhood.

The astrometric observations reported here began as part of the NOAO Surveys Program in 1999 and continued on the CTIO $0.9 \mathrm{~m}$ via the SMARTS Consortium starting in 2003. We gratefully acknowledge support from the National Science Foundation (grants AST 05-07711, AST 09-08402, and AST 14-12026), NASA's Space Interferometry Mission, and Georgia State University, which together have made this long-term effort possible. We also thank the members of the SMARTS Consortium, who enable the operations of the small

\footnotetext{
13 This star has an L4 companion (Radigan et al. 2008) that does not have PanSTARRS photometry, so it is not shown in the plot.
}

telescopes at CTIO, as well as the supporting observers at CTIO, specifically Edgardo Cosgrove, Arturo Gómez, Alberto Miranda, and Joselino Vásquez.

The HST-FGS observations were supported under program 11943 by NASA through grants from the Space Telescope Science Institute, which is operated by the Association of Universities for Research in Astronomy, Inc., under NASA contract NAS5-26555.

This research has made use of the SIMBAD database, operated at CDS, Strasbourg, France. This publication makes use of data products from the Two Micron All Sky Survey, which is a joint project of the University of Massachusetts and the Infrared Processing and Analysis Center/California Institute of Technology, funded by the National Aeronautics and Space Administration and the National Science Foundation. This work also has made use of data from the European Space Agency (ESA) mission Gaia (https://www.cosmos.esa. int/gaia), processed by the Gaia Data Processing and Analysis Consortium (DPAC, https://www.cosmos.esa.int/web/gaia/ dpac/consortium). Funding for the DPAC has been provided by national institutions, particularly the institutions participating in the Gaia Multilateral Agreement.

The Pan-STARRS1 Surveys (PS1) and the PS1 public science archive, which were used for this study, have been made possible through contributions by the Institute for Astronomy, the University of Hawaii, the Pan-STARRS Project Office, the Max Planck Society and its participating institutes, the Max Planck Institute for Astronomy, Heidelberg, the Max Planck Institute for Extraterrestrial Physics, Garching, The Johns Hopkins University, Durham University, the University of Edinburgh, the Queen's University Belfast, the Harvard-Smithsonian Center for Astrophysics, the Las Cumbres Observatory Global Telescope Network Incorporated, the National Central University of Taiwan, the Space Telescope Science Institute, the National Aeronautics and Space Administration under Grant No. NNX08AR22G issued through the Planetary Science Division of the NASA Science Mission Directorate, the National Science Foundation through Grant No. AST-1238877, the University of Maryland, Eotvos Lorand University (ELTE), the Los Alamos National Laboratory, and the Gordon and Betty Moore Foundation.

\section{ORCID iDs}

Wei-Chun Jao (iD https://orcid.org/0000-0003-0193-2187 John P. Subasavage (iD https://orcid.org/0000-0001-5912-6191 Adric R. Riedel (1) https://orcid.org/0000-0003-1645-8596 Michele L. Silverstein (1) https://orcid.org/0000-00032565-7909

\section{References}

Alonso-Floriano, F. J., Morales, J. C., Caballero, J. A., et al. 2015, A\&A, 577, A128

Arifyanto, M. I., Fuchs, B., Jahreiß, H., \& Wielen, R. 2005, A\&A, 433, 911

Benedict, G. F., Henry, T. J., Franz, O. G., et al. 2016, AJ, 152, 141

Berger, D. H., Gies, D. R., McAlister, H. A., et al. 2006, ApJ, 644, 475

Bergeron, P., Ruiz, M. T., \& Leggett, S. K. 1997, ApJS, 108, 339

Bessell, M. S. 1982, PASAu, 4, 417

Bidelman, W. P. 1985, ApJS, 59, 197

Blake, C. H., Charbonneau, D., \& White, R. J. 2010, ApJ, 723, 684 Boyajian, T. S., McAlister, H. A., Baines, E. K., et al. 2008, ApJ, 683, 424 Boyajian, T. S., von Braun, K., van Belle, G., et al. 2012, ApJ, 757, 112 Burgasser, A. J., Cruz, K. L., \& Kirkpatrick, J. D. 2007, ApJ, 657, 494 Burgasser, A. J., Kirkpatrick, J. D., Burrows, A., et al. 2003, ApJ, 592, 1186 Burgasser, A. J., Vrba, F. J., Lépine, S., et al. 2008, ApJ, 672, 1159 
Carney, B. W., Latham, D. W., Laird, J. B., \& Aguilar, L. A. 1994, AJ, 107,2240

Cayrel de Strobel, G., Soubiran, C., \& Ralite, N. 2001, A\&A, 373, 159

Chamberlain, J. W., \& Aller, L. H. 1951, ApJ, 114, 52

Costa, E., Méndez, R. A., Jao, W.-C., et al. 2005, AJ, 130, 337

Cruz, K. L., \& Reid, I. N. 2002, AJ, 123, 2828

Cushing, M. C., \& Vacca, W. D. 2006, AJ, 131, 1797

Cutispoto, G., Pastori, L., Pasquini, L., et al. 2002, A\&A, 384, 491

Dahn, C. C., Harris, H. C., Levine, S. E., et al. 2008, ApJ, 686, 548

Deacon, N. R., Hambly, N. C., \& Cooke, J. A. 2005, A\&A, 435, 363

Dieterich, S. B., Henry, T. J., Jao, W.-C., et al. 2014, AJ, 147, 94

Digby, A. P., Hambly, N. C., Cooke, J. A., Reid, I. N., \& Cannon, R. D. 2003, MNRAS, 344, 583

Dittmann, J. A., Irwin, J. M., Charbonneau, D., \& Berta-Thompson, Z. K. 2014, ApJ, 784, 156

Faherty, J. K., Burgasser, A. J., Walter, F. M., et al. 2012, ApJ, 752, 56

Farihi, J., Hoard, D. W., \& Wachter, S. 2010, ApJS, 190, 275

Flewelling, H. A., Magnier, E. A., Chambers, K. C., et al. 2016, arXiv:1612. 05243

Friedrich, S., Koester, D., Christlieb, N., Reimers, D., \& Wisotzki, L. 2000, A\&A, 363, 1040

Gagné, J., Lafrenière, D., Doyon, R., Malo, L., \& Artigau, É. 2014, ApJ, 783,121

Gaia Collaboration, Brown, A. G. A., Vallenari, A., et al. 2016, A\&A, 595, A2 Giclas, H. L. 1979, NASA STI/Recon Tech. Rep. N, 80

Giclas, H. L., Burnham, R., \& Thomas, N. G. 1971, Lowell proper motion survey Northern Hemisphere. The G numbered stars. 8991 stars fainter than magnitude 8 with motions $>0$ "! 26/year (Flagstaff, Arizona: Lowell Observatory)

Gizis, J. E. 1997, AJ, 113, 806

Gizis, J. E., Monet, D. G., Reid, I. N., et al. 2000, AJ, 120, 1085

Gizis, J. E., \& Reid, I. N. 1997, PASP, 109, 849

Gizis, J. E., \& Reid, I. N. 1999, AJ, 117, 508

Gizis, J. E., Troup, N. W., \& Burgasser, A. J. 2011, ApJL, 736, L34

Goldberg, D., Mazeh, T., Latham, D. W., et al. 2002, AJ, 124, 1132

Haakonsen, C. B., \& Rutledge, R. E. 2009, ApJS, 184, 138

Hambly, N. C., Henry, T. J., Subasavage, J. P., Brown, M. A., \& Jao, W.-C. 2004, AJ, 128, 437

Hawley, S. L., Gizis, J. E., \& Reid, I. N. 1996, AJ, 112, 2799

Henry, T. J., Franz, O. G., Wasserman, L. H., et al. 1999, ApJ, 512, 864

Henry, T. J., Jao, W.-C., Subasavage, J. P., et al. 2006, AJ, 132, 2360

Henry, T. J., Subasavage, J. P., Brown, M. A., et al. 2004, AJ, 128, 2460

Henry, T. J., Walkowicz, L. M., Barto, T. C., \& Golimowski, D. A. 2002, AJ, 123,2002

Høg, E., Fabricius, C., Makarov, V. V., et al. 2000, A\&A, 355, L27

Hosey, A. D., Henry, T. J., Jao, W.-C., et al. 2015, AJ, 150, 6

Jao, W.-C., Henry, T. J., Beaulieu, T. D., \& Subasavage, J. P. 2008, AJ, 136,840

Jao, W.-C., Henry, T. J., Subasavage, J. P., et al. 2005, AJ, 129, 1954

Jao, W.-C., Henry, T. J., Subasavage, J. P., et al. 2011, AJ, 141, 117

Jao, W.-C., Mason, B. D., Hartkopf, W. I., Henry, T. J., \& Ramos, S. N. 2009, AJ, 137, 3800

Jao, W.-C., Nelan, E. P., Henry, T. J., Franz, O. G., \& Wasserman, L. H. 2016, AJ, 152, 153

Kirkpatrick, J. D., Cushing, M. C., Gelino, C. R., et al. 2011, ApJS, 197, 19

Kirkpatrick, J. D., Kellogg, K., Schneider, A. C., et al. 2016, ApJS, 224, 36

Koren, S. C., Blake, C. H., Dahn, C. C., \& Harris, H. C. 2016, AJ, 151, 57

Kuiper, G. P. 1939, ApJ, 89, 548
Latham, D. W., Stefanik, R. P., Torres, G., et al. 2002, AJ, 124, 1144

Lépine, S., \& Bongiorno, B. 2007, AJ, 133, 889

Lépine, S., Rich, R. M., \& Shara, M. M. 2003a, ApJL, 591, L49

Lépine, S., Rich, R. M., \& Shara, M. M. 2003b, AJ, 125, 1598

Lépine, S., Rich, R. M., \& Shara, M. M. 2007, ApJ, 669, 1235

Lépine, S., \& Shara, M. M. 2005, AJ, 129, 1483

Li, J. Z., Hu, J. Y., \& Chen, W. P. 2000, A\&A, 356, 157

López-Morales, M. 2007, ApJ, 660, 732

Luyten, W. J. 1979, LHS Catalog. A catalogue of stars with proper motions exceeding 0"5 annually (2nd ed.; Minneapolis, MN: Univ. Minnesota Press)

Monteiro, H., Jao, W.-C., Henry, T., Subasavage, J., \& Beaulieu, T. 2006, ApJ, 638,446

Mould, J. R. 1976, A\&A, 48, 443

Newton, E. R., Irwin, J., Charbonneau, D., et al. 2016, ApJ, 821, 93

Perryman, M. A. C., Lindegren, L., Kovalevsky, J., et al. 1997, A\&A, 323, L49

Pokorny, R. S., Jones, H. R. A., \& Hambly, N. C. 2003, A\&A, 397, 575

Radigan, J., Lafrenière, D., Jayawardhana, R., \& Doyon, R. 2008, ApJ, 689, 471

Rebull, L. M., Stauffer, J. R., Bouvier, J., et al. 2016, AJ, 152, 113

Reid, I. N. 2003, AJ, 126, 2449

Reid, I. N., Cruz, K. L., Allen, P., et al. 2004, AJ, 128, 463

Reid, I. N., Cruz, K. L., \& Allen, P. R. 2007, AJ, 133, 2825

Reid, I. N., \& Gizis, J. E. 2005, PASP, 117, 676

Reylé, C., Scholz, R.-D., Schultheis, M., Robin, A. C., \& Irwin, M. 2006, MNRAS, 373, 705

Riedel, A. R., Murphy, S. J., Henry, T. J., et al. 2011, AJ, 142, 104

Rodgers, A. W., \& Eggen, O. J. 1974, PASP, 86, 742

Ryan, S. G., \& Norris, J. E. 1991, AJ, 101, 1835

Savcheva, A. S., West, A. A., \& Bochanski, J. J. 2014, ApJ, 794, 145

Schilbach, E., Röser, S., \& Scholz, R.-D. 2009, A\&A, 493, L27

Schmidt, M. 1975, ApJ, 202, 22

Scholz, R.-D., Lehmann, I., Matute, I., \& Zinnecker, H. 2004a, A\&A, 425, 519

Scholz, R.-D., Lodieu, N., \& McCaughrean, M. J. 2004b, A\&A, 428, L25

Scholz, R.-D., Meusinger, H., \& Jahreiß, H. 2005, A\&A, 442, 211

Ségransan, D., Kervella, P., Forveille, T., \& Queloz, D. 2003, A\&A, 397, L5

Skrutskie, M. F., et al. 2006, AJ, 131, 1163

Smart, R. L., Ioannidis, G., Jones, H. R. A., Bucciarelli, B., \& Lattanzi, M. G. 2010, A\&A, 514, A84

Soubiran, C., Le Campion, J.-F., Cayrel de Strobel, G., \& Caillo, A. 2010, A\&A, 515, A111

Sperauskas, J., Bartašiūtė, S., Boyle, R. P., et al. 2016, A\&A, 596, A116

Subasavage, J. P., Henry, T. J., Hambly, N. C., et al. 2005, AJ, 130, 1658

Torres, G., Andersen, J., \& Giménez, A. 2010, A\&ARv, 18, 67

van Altena, W. F., Lee, J. T., \& Hoffleit, D. 1995, The General Catalogue of Trigonometric Stellar Parallaxes (4th ed.; New Haven: Yale Univ. Obs.) http://adsabs.harvard.edu/abs/1995yCat.1174....0V

van Leeuwen, F. 2007, A\&A, 474, 653

Walker, A. R. 1983, SAAOC, 7, 106

Weis, E. W. 1988, AJ, 96, 1710

West, A. A., Hawley, S. L., Walkowicz, L. M., et al. 2004, AJ, 128, 426

White, R. J., Gabor, J. M., \& Hillenbrand, L. A. 2007, AJ, 133, 2524

Winters, J. G., Henry, T. J., Jao, W.-C., et al. 2011, AJ, 141, 21

Winters, J. G., Henry, T. J., Lurie, J. C., et al. 2015, AJ, 149, 5

Winters, J. G., Sevrinsky, R. A., Jao, W.-C., et al. 2017, AJ, 153, 14

Woolf, V. M., \& Wallerstein, G. 2005, MNRAS, 356, 963

Wright, E. L., Kirkpatrick, J. D., Gelino, C. R., et al. 2014, AJ, 147, 61

Zhang, Z. H., Pinfield, D. J., Burningham, B., et al. 2013, MNRAS, 434, 1005

Zhong, J., Lépine, S., Hou, J., et al. 2015, AJ, 150, 42 